\title{
Quantum Amplification by Superradiant Emission of Radiation
}

\author{
Anatoly A. Svidzinsky, ${ }^{1,2}$ Luqi Yuan, ${ }^{1,2}$ and Marlan O. Scully ${ }^{1,2,3}$ \\ ${ }^{1}$ Texas A\&M University, College Station, Texas 77843, USA \\ ${ }^{2}$ Princeton University, Princeton, New Jersey 08544, USA \\ ${ }^{3}$ Baylor University, Waco, Texas 76706, USA
}

(Received 18 June 2013; revised manuscript received 31 July 2013; published 8 October 2013)

\begin{abstract}
A laser generates light through stimulated emission of radiation and requires population inversion. Quantum interference can yield lasing without inversion. However, such phase-sensitive quantum amplification still requires some atomic population in the excited state. Here, we present a new kind of quantum amplifier based on collective superradiant emission which does not need any population in the excited state. We show that parametric resonance between the driving (e.g., infrared) field and collective superradiant oscillations of the atomic polarization can yield light amplification at high (e.g., XUV) frequencies. To achieve gain, one must suppress a time-dependent Stark shift caused by the driving field. The resulting superradiant amplifier is many orders of magnitude more efficient than the usual nonlinear multiphoton excitation and holds promise for a new kind of generator of highfrequency coherent radiation. In addition to a detailed analytical analysis, confirmed by numerical simulations, we provide a physically appealing explanation of the quantum amplification by superradiant emission of radiation (QASER) operation in terms of coupled classical oscillators. We also present an experiment that demonstrates the QASER amplification mechanism in an electronic circuit, which, to the best of our knowledge, is the first experimental demonstration of the difference combination resonance.
\end{abstract}

DOI: 10.1103/PhysRevX.3.041001

\section{INTRODUCTION}

Phase matching between intense classical laser fields is an essential feature of nonlinear optics. But with the advent of giant nonlinearities made possible by electromagnetically included transparency and ultraslow light, we were asked the following question: Can we have phase matching at the single-photon level? Our answer is yes, and this interesting question stimulated much of the recent work on single-photon superradiance, which focuses on collective, virtual, and nonlocal effects [1-15]. In particular, in the Dicke model of $N$ two-level $(|a\rangle$ and $|b\rangle)$ atoms [16], in a small atomic sample of radius $R$ much less than the radiation wavelength $\lambda$, the symmetric state with only one atom excited,

$$
\left|\Psi_{s}\right\rangle=\frac{1}{\sqrt{N}} \sum_{j=1}^{N}\left|b_{1}, b_{2} \ldots a_{j} \ldots b_{N}\right\rangle
$$

decays to the ground state $\left|b_{1}, b_{2} \ldots b_{j} \ldots b_{N}\right\rangle$ at the rate $\Gamma_{s}=N \gamma$, where $\gamma$ is the single-atom spontaneous decay rate. Dicke called this "the greatest radiation anomaly" of superradiance. However, if $R \gg \lambda$, the state (1) will trap light, decreasing the emission rate.

Published by the American Physical Society under the terms of the Creative Commons Attribution 3.0 License. Further distribution of this work must maintain attribution to the author(s) and the published article's title, journal citation, and DOI.
Nevertheless, it is possible to prepare a phased (timed) state excited by a photon of wave vector $\mathbf{k}_{0}$ and frequency $\nu_{0}[1]$,

$$
\left|\Psi_{p}\right\rangle=\frac{1}{\sqrt{N}} \sum_{j=1}^{N} e^{i \mathbf{k}_{0} \cdot \mathbf{r}_{j}}\left|b_{1}, b_{2}, \ldots a_{j} \ldots b_{N}\right\rangle,
$$

which, to a good approximation, decays with the enhanced rate $\Gamma_{p} \cong N \gamma \lambda^{2} / R^{2}$. Physically, the phase factors in Eq. (2), $\quad \exp \left(i \mathbf{k}_{0} \cdot \mathbf{r}_{j}\right)=\exp \left(i \nu_{0} \Delta t_{j}\right), \quad$ where $\Delta t_{j}=$ $\hat{k}_{0} \cdot \mathbf{r}_{j} / c$, arise from the fact that atoms at the front of the sample are excited first and atoms further downstream at position $\mathbf{r}_{j}$ are excited later [1].

However, when the cloud radius is large compared to the superradiant pulse length $c / \Gamma_{p}$, the situation becomes even more interesting $[3,7,15]$. In such a case, the emitted photon is reabsorbed and reemitted many times, as shown in Fig. 1. This limit is the essence of cavity QED, where photons in a cavity resonantly interact with a single atom. If initially there are $N_{\mathrm{ph}}$ photons and the atom is in the ground state, then the probability of finding an excited atom oscillates as

$$
P_{\text {cavity }}=\sin ^{2}\left(\frac{\wp}{\hbar} \sqrt{\frac{\hbar \nu N_{\mathrm{ph}}}{\epsilon_{0} V_{\mathrm{ph}}}} t\right)
$$

where $\wp$ is the atomic transition matrix element, $\hbar \nu$ is the photon energy, and $V_{\mathrm{ph}}$ is the cavity volume. However, in the present case, when a single photon interacts with a 


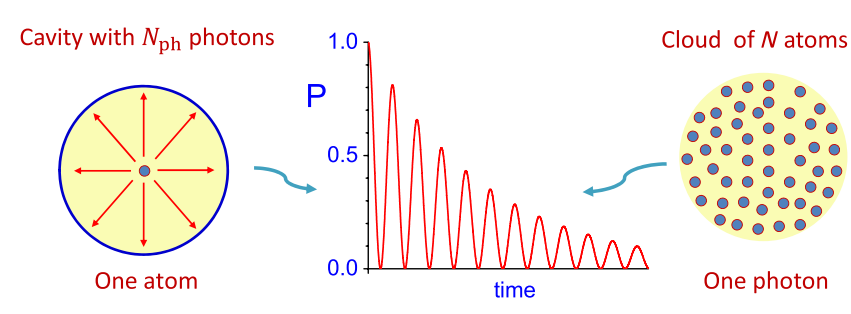

FIG. 1. For a very large cloud, the photon is reabsorbed and reemitted many times, and the atomic state oscillates with a frequency that goes as $\sqrt{N}$. This behavior is to be compared to the cavity QED scenario in which an atom is cycled between the ground and excited states with a frequency that goes as $\sqrt{N_{\mathrm{ph}}}$, where $N_{\text {ph }}$ is the number of photons in the cavity. $\mathrm{P}$ is the probability that an atom is excited.

large cloud of $N$ atoms in free space, the probability of finding an excited atom is $[3,15]$

$$
P_{\text {cloud }}=\sin ^{2}\left(\frac{\wp}{\hbar} \sqrt{\frac{\hbar \nu N}{\epsilon_{0} V}} t\right)
$$

where the cavity volume $V_{\mathrm{ph}}$ and the photon number $N_{\mathrm{ph}}$ are replaced by the cloud volume $\mathcal{V}$ and the number of atoms $N$.

The following question naturally arises: The stimulated emission implied by Eq. (3) is the basis for the laser. Does Eq. (4) suggest a corresponding new but different (since collective spontaneous emission is different from stimulated emission) source of coherent radiation? As we shall see, the answer is yes, modulo certain somewhat subtle considerations. In particular, we show here, for the first time, that by utilizing collective superradiant emission, we can generate coherent light at high frequency in the UV or x-ray bands by driving the atomic system with a low-frequency (e.g., infrared) source. This process involves a phase-dependent quantum gain, as in the case of lasing without inversion (LWI), which is another example of quantum amplification. We call the present device that generates high-frequency light through quantum amplification by superradiant emission of radiation the "QASER."

To set the stage, let us first recall the equations that describe conventional lasing and superradiance. We consider a medium composed of two-level $(|a\rangle$ and $|b\rangle)$ atoms, with the population of the excited and ground states being $\rho_{a a}$ and $\rho_{b b}$, respectively. We are interested in the evolution of a weak laser (superradiant) pulse at the atomic transition frequency $\omega_{a b}$ that propagates along the $z$ axis. Since the pulse is weak, the populations $\rho_{a a}$ and $\rho_{b b}$ can be treated as constant. In the semiclassical approach, evolution of the envelope $\Omega_{s}$ of the superradiant pulse is described by the Maxwell-Schrödinger equations

$$
\left(\frac{\partial}{\partial t}+c \frac{\partial}{\partial z}\right) \Omega_{s}=i \Omega_{a}^{2} \rho_{a b}^{s},
$$

$$
\dot{\rho}_{a b}^{s}=-\gamma_{\mathrm{tot}} \rho_{a b}^{s}+i \Omega_{s}\left(\rho_{b b}-\rho_{a a}\right)
$$

where

$$
\Omega_{a}=\sqrt{\frac{3 n \lambda_{a b}^{2} \gamma c}{8 \pi}}
$$

is a collective atomic frequency, $n=N / \mathcal{V}$ is the atomic density, $\lambda_{a b}$ is the wavelength of the $a-b$ transition, $\gamma$ is the spontaneous decay rate of a single atom, $\rho_{a b}^{s}$ is slowly varying envelope of atomic coherence, $\Omega=\wp_{a b} \cdot \mathbf{E} / \hbar$ is the Rabi frequency corresponding to electric field $\mathbf{E}$, $\wp_{a b}=\langle a|e \mathbf{r}| b\rangle$ is the matrix element of the electric dipole moment, and $\gamma_{\text {tot }}$ is the decoherence rate. $\Omega_{a}$ is the collective frequency with which the resonant pulse is absorbed and reemitted by the medium $[3,15,17]$.

Taking the time derivative of both sides of Eq. (5) and using Eq. (6), we obtain for the pulse envelope $\Omega_{s}$ the following linear equation with constant coefficients:

$\left(\frac{\partial}{\partial t}+\gamma_{\mathrm{tot}}\right)\left(\frac{\partial}{\partial t}+c \frac{\partial}{\partial z}\right) \Omega_{s}+\Omega_{a}^{2}\left(\rho_{b b}-\rho_{a a}\right) \Omega_{s}=0$.

One can look for the solution of Eq. (8) in the form

$$
\Omega_{s}=A \exp (i k z-i \nu t),
$$

where $A$ is a constant, $\nu$ is the envelope frequency which is essentially the detuning of the superradiant field from the atomic transition frequency, and $k$ is the envelope wave number. If we treat $k$ as real, then the imaginary part of $\nu$ gives gain (absorption) per unit time $G=\operatorname{Im}(\nu)$ for a mode with wave number $k$ and $\Omega_{s} \propto e^{G t}$. Plugging (9) into Eq. (8) yields

$\nu=\frac{1}{2}\left[-i \gamma_{\mathrm{tot}}+c k \pm \sqrt{\left(i \gamma_{\mathrm{tot}}+c k\right)^{2}+4 \Omega_{a}^{2}\left(\rho_{b b}-\rho_{a a}\right)}\right]$,

which, for the mode with $k \ll \Omega_{a} / c$, gives the gain

$$
\begin{aligned}
G=\Omega_{a} \sqrt{\rho_{a a}-\rho_{b b}}, & G \gg \gamma_{\mathrm{tot}}, \\
G=\frac{\Omega_{a}^{2}}{\gamma_{\mathrm{tot}}}\left(\rho_{a a}-\rho_{b b}\right), & G \ll \gamma_{\mathrm{tot}} .
\end{aligned}
$$

If there is population inversion, $\rho_{a a}>\rho_{b b}$, then gain is positive and the weak seed field grows exponentially with time. The limit $G \gg \gamma_{\text {tot }}$ corresponds to superradiance in which the pulse evolves on a time scale much faster than the single-atom decay time. In such a limit, $G \sim \sqrt{n}$. On the other hand, if $\rho_{a a}=0$ (all population is in the ground state) and $\gamma_{\text {tot }}=0$, then Eq. (11) yields imaginary $G$, which means $\Omega_{s}$ oscillates with collective frequency $\Omega_{a}$. Such oscillations describe collective absorption and superradiant reemission of light by the atomic system [3,15,17].

During the last two decades, lasing without inversion (LWI) has been discussed in the literature for various schemes [18-21]. Typically, LWI is achieved by quantum interference in emission or absorption channels. Such interference 
appears in systems with coherence created by an external source. However, LWI models require nonzero population of atoms in the excited state.

Here, we go further and show that light amplification (gain) can be obtained even if initially $\rho_{a a}=0$. This can occur in the superradiant regime because of a resonance between the driving-field frequency $\nu_{d}$ and collective polarization oscillation at $\Omega_{a}$. Before going into details and lengthy calculations, we next motivate the present gain mechanism in a simple way. Let us assume that in Eq. (8) population is periodically modulated with frequency $\nu_{d}$ such that

$$
\rho_{b b}-\rho_{a a}=1-\delta \cos \left(2 \nu_{d} t\right),
$$

where $\delta \ll 1$ is a small modulation amplitude. Writing the slowly varying envelope $\Omega_{s}(t, z)$ in the form

$$
\Omega_{s}(t, z)=\Omega_{s}(t) \exp \left[i k\left(z-\frac{c t}{2}\right)\right]
$$

where $k$ is the envelope wave number, and neglecting decoherence, we obtain the simple equation for $\Omega_{s}(t)$,

$$
\ddot{\Omega}_{s}+\tilde{\Omega}_{a}^{2}\left[1-\tilde{\delta} \cos \left(2 \nu_{d} t\right)\right] \Omega_{s}=0,
$$

where

$$
\tilde{\Omega}_{a}^{2}=\Omega_{a}^{2}+\frac{c^{2} k^{2}}{4}
$$

and $\tilde{\delta}=\Omega_{a}^{2} \delta / \tilde{\Omega}_{a}^{2}$. Equation (15) is known as the Mathieu equation [22], which yields exponentially growing oscillations in the vicinities of parametric resonances when $\nu_{d} \approx \tilde{\Omega}_{a} / m, m=1,2,3, \ldots$ [see Fig. 2(a)]. In particular, for the (strongest) first-order resonance $\left(\nu_{d}=\tilde{\Omega}_{a}\right)$, gain per unit time is given by [23]

(a)
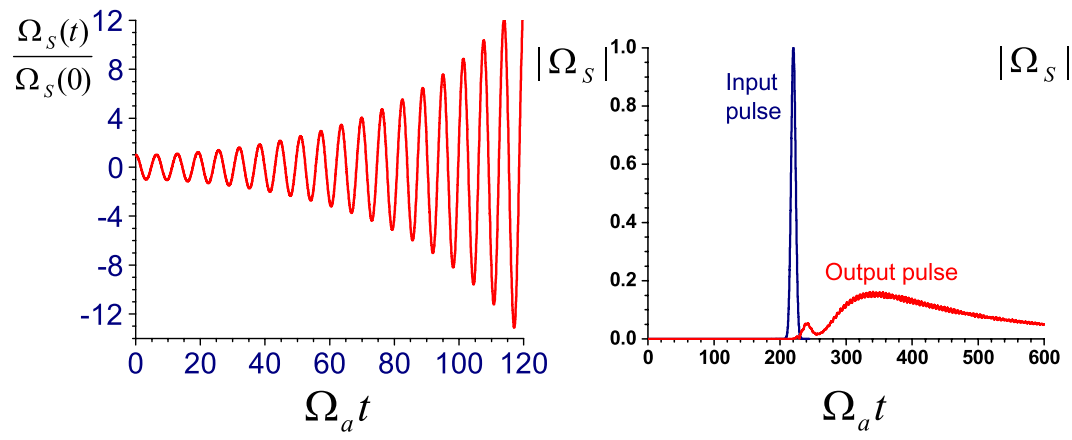

(b)

$$
G=\frac{\tilde{\delta} \cdot \tilde{\Omega}_{a}}{4}=\frac{\delta \cdot \Omega_{a}^{2}}{4 \nu_{d}} .
$$

Equation (16) implies that the wave number of the exponentially growing mode is

$$
k= \pm \frac{2}{c} \sqrt{\nu_{d}^{2}-\Omega_{a}^{2}} .
$$

Hence, if the driving-field frequency obeys the condition

$$
\nu_{d}>\Omega_{a},
$$

there is a mode that is in parametric resonance with $\nu_{d}$. Such a mode grows exponentially with gain given by Eq. (17). In other words, the system yields gain at the high atomic frequency with no population in the excited state. For example, for the $4{ }^{1} \mathrm{P} \rightarrow{ }^{1} \mathrm{~S}$ transition of the $\mathrm{He}$ atom, $\lambda_{a b}=52.2 \mathrm{~nm}$ and spontaneous decay rate is $\gamma=$ $2.4 \times 10^{8} \mathrm{~s}^{-1}$. Then, for atomic density $n=10^{20} \mathrm{~cm}^{-3}$, Eq. (7) shows that the collective atomic frequency lies in the infrared band $\Omega_{a}=0.48 \times 10^{14} \mathrm{~s}^{-1}$ while the atomic frequency is in the XUV range $\omega_{a b}=0.36 \times 10^{17} \mathrm{~s}^{-1}$.

A key point of this article is that we have discovered a new mechanism of atomic excitation by means of collective resonance. This mechanism is different from the wellknown multiphoton resonant excitation of an atom, which occurs when the driving-field frequency $\nu_{d}=\omega_{a b} / m$, $m=1,2, \ldots$. Ordinary multiphoton resonant excitation is a single-atom phenomenon rather than a collective effect. In the present case, there is a resonance between $\nu_{d}$ and collective oscillations, which occurs for $\nu_{d} \geqslant \Omega_{a}$.

Excited-state population $\rho_{a a}$ grows with time differently in the two cases. For multiphoton resonance and a weak driving field, we have for $\rho_{a a} \ll 1[24]$

$$
\rho_{a a} \approx\left(\frac{\Omega_{d}}{\omega_{a b}}\right)^{2 m-2}\left(\Omega_{d} t\right)^{2},
$$

FIG. 2. (a) Superradiant field as a function of time, obtained by numerical solution of Eq. (15) with $\tilde{\delta}=0.1, \nu_{d}=\tilde{\Omega}_{a}$ and initial condition $\dot{\Omega}_{l}(0)=0$. (b) Input and output superradiant pulse $\Omega_{s}$ as a function of time after it propagates through the sample of length $L=100 c / \Omega_{a}$. Atoms are driven by the electric field with Rabi frequency (26) and by the magnetic field (33), which compensates the atomic Stark shift. The superradiant pulse is sent in the same direction as the driving field. Plots are obtained by numerical solution of Eqs. (B65)-(B69), with $\omega_{a b}=5.2 \nu_{d}, \nu_{d}=0.64 \Omega_{a}$, and $\Omega_{d}=\Omega_{a}$. Units of $\Omega_{s}$ are arbitrary. (c) The same as in Fig. 2(b) but for the backward-propagating superradiant pulse. 
where $\Omega_{d}$ is the Rabi frequency of the driving field. However, the present work is based on the collective parametric resonance and, as we show below, the growth is exponential (see Appendix D for details):

$$
\rho_{a a} \approx\left|\rho_{a b}(0)\right|^{2} \exp \left(\frac{\sqrt{2}}{3} \frac{\Omega_{d}^{2}}{\omega_{a b}^{2}} \Omega_{a} t\right),
$$

where $\rho_{a b}(0)$ is the initial-seed atomic coherence (produced by the seed XUV pulse).

Finally, excitation by multiphoton resonance yields emission of light at high atomic frequency $\omega_{a b}$ in the direction of the driving field. In contrast, in the present case, the emission occurs in the opposite direction.

\section{QUANTUM GAIN BY COLLECTIVE SUPERRADIANT RESONANCE}

Next, we present a rigorous analysis that demonstrates the possibility of having quantum gain by collective superradiance. We consider the same medium composed of two-level ( $a$ and $b$ ) atoms with frequency $\omega_{a b}$, which are modulated by a coherent driving field propagating along the $z$ axis and having Rabi frequency $\Omega^{\text {drive }}(t, z)$. We suppose that the driving-field frequency $\nu_{d} \ll \omega_{a b}$ and $\left|\Omega^{\text {drive }}\right| \ll \omega_{a b}$, so that modulation is weak.

In Appendix A, we derive the propagation equation for the weak high-frequency pulse $\Omega_{s}$ and obtain

$$
\begin{aligned}
& \left(\frac{\partial}{\partial t}+i \Delta \omega_{a b}(t)+\frac{2 \Omega^{\text {drive }} \dot{\Omega}^{\text {drive* }}}{\omega_{a b}^{2}}\right)\left(c \frac{\partial}{\partial z}+\frac{\partial}{\partial t}\right) \Omega_{s} \\
& \quad+\Omega_{a}^{2}\left(1-2 \rho_{a a}^{\text {drive }}-\frac{2\left|\Omega^{\text {drive }}\right|^{2}}{\omega_{a b}^{2}}-i \frac{4 \Omega^{\text {drive }}}{\omega_{a b}} \operatorname{Im}\left[\rho_{a b}^{\text {drive }}\right]\right) \Omega_{s} \\
& =0,
\end{aligned}
$$

where

$$
\Delta \omega_{a b}(t)=\frac{2\left|\Omega^{\mathrm{drive}}(t, z)\right|^{2}}{\omega_{a b}}
$$

is the (undesirable) time-dependent Stark shift of the atomic transition frequency proportional to the instantaneous intensity of the driving field.

Equation (22) shows that the driving field produces several effects that contribute to evolution of the superradiant pulse $\Omega_{s}$. The time-dependent Stark shift $\Delta \omega_{a b}(t)$ caused by the drive is the leading contribution since it is proportional to $1 / \omega_{a b}$. Such a term suppresses collective resonance, which is governed by the next-order corrections. As a result, in order to obtain gain at the high atomic frequency, one should compensate the unwanted Stark shift. This can be done in several ways, which we discuss next.

For example, in the present two-level atom model, the time-dependent Stark shift can be eliminated if the driving field is nearly circularly polarized so that the Rabi frequency has the form

$$
\Omega^{\text {drive }}(t, z)=\Omega_{1} e^{i \nu_{d} t-i k_{d} z}+\Omega_{2} e^{-i \nu_{d} t+i k_{d} z},
$$

where $\Omega_{1}$ and $\Omega_{2}$ are real constants and $k_{d}$ is the wave number of the driving field. Such a situation can be realized for the elliptically polarized driving field $\mathbf{E}^{\text {drive }}$ if the dipole matrix element of the two-level atom is

$$
\mathbf{d}_{a b}=d_{x} \hat{x}+i d_{y} \hat{y},
$$

where $d_{x}$ and $d_{y}$ are real. This is, e.g., the case if the excited state $a$ corresponds to a $p_{x}+i p_{y}$ atomic orbital and $b$ is an $s$ state.

As we show in Appendix B 1, for a uniform driving field $\left(k_{d}=0\right)$ and a certain ratio of $\Omega_{2} / \Omega_{1}$, the Stark shift vanishes. Namely, to suppress the unwanted Stark shift, we should choose

$$
\frac{\Omega_{2}}{\Omega_{1}}=\frac{\Omega_{a}^{2}}{2 \nu_{d} \omega_{a b}} .
$$

Under such conditions, Eq. (22) yields exponential grow of the seed laser pulse, with gain $G=\Omega_{a} \Omega_{1}^{2} / 2 \omega_{a b}^{2}$. This example demonstrates the possibility of having gain in a simple model in which the Stark shift is compensated by driving two-level atoms with a field of certain polarization.

In the general case, the driving field and the growing XUV modes depend on spatial coordinates. In Appendix B 2 , we explore such a situation for a different model in which atoms are driven by linearly polarized light propagating along the $z$ axis,

$$
\Omega^{\text {drive }}(t, z)=\Omega_{d} \cos \left(\nu_{d} t-k_{d} z\right),
$$

and the time-dependent Stark shift is compensated by applying an additional magnetic field $H(t)$ with Rabi frequency $\Omega_{H}(t)=\mu H / \hbar$. In this model, the magnetic field produces a time-dependent Zeeman shift $\Omega_{H}(t)$ of the level $a$, which can compensate the unwanted Stark shift of the $a-b$ transition frequency. We find that if the Stark shift is compensated by a magnetic field, then the XUV pulse grows exponentially, provided that

$$
c k_{d}<\nu_{d}
$$

and

$$
\nu_{d}^{2} \geq \frac{\nu_{d}}{\nu_{d}-c k_{d}} \Omega_{a}^{2} .
$$

Maximum gain per unit time is

$$
G=\frac{\sqrt{\nu_{d}^{2}-c k_{d} \nu_{d}}}{2 \nu_{d}-c k_{d}} \frac{\Omega_{a}}{2} \frac{\Omega_{d}^{2}}{\omega_{a b}^{2}},
$$

while gain per unit length is given by

$$
G_{\mathrm{L}} \approx \frac{\left|\nu_{d}-c k_{d}\right|}{2 c} \frac{\Omega_{d}^{2}}{\omega_{a b}^{2}} .
$$

In particular, Eqs. (29) and (30) show that there is no gain in the direction of the driving field (when $c k_{d}=\nu_{d}$ ). 
However, there is gain in the backward direction (when $c k_{d}=-\nu_{d}$ ), provided that $\nu_{d}>\Omega_{a} / \sqrt{2}$, namely,

$$
G=\frac{\Omega_{a}}{3 \sqrt{2}} \frac{\Omega_{d}^{2}}{\omega_{a b}^{2}}
$$

and

$$
G_{\mathrm{L}} \approx \frac{\nu_{d}}{c} \frac{\Omega_{d}^{2}}{\omega_{a b}^{2}} .
$$

Finally, we investigate numerically how the weak XUV pulse evolves, assuming that atoms are driven by linearly polarized light (26) with $k_{d}=\nu_{d} / c$ and the Stark shift is compensated by applying an additional magnetic field (for details, see Appendix B 3). The field (26) is turned on adiabatically. In calculations, we take $\omega_{a b}=5.2 \nu_{d}$, $\nu_{d}=0.64 \Omega_{a}$, and $\Omega_{d}=\Omega_{a}$. We send a weak XUV pulse of Gaussian shape into a sample of length $L=100 c / \Omega_{a}$ and calculate how it evolves, provided that the Stark shift is compensated by choosing $\Omega_{H}$ as

$$
\Omega_{H}(t, z)=\frac{2}{\omega_{a b}}\left[\Omega^{\text {drive }}(t, z)\right]^{2} .
$$

The numerical results are shown in Fig. 2(b) (for an $\mathrm{XUV}$ pulse propagating in the same direction as the driving field) and Fig. 2(c) for backward propagation. Figure 2(b) demonstrates that there is no gain in the forward direction. However, the XUV pulse grows in the backward direction, and its intensity increases by several orders of magnitude after propagation through the sample, in agreement with our analytical findings. We also performed numerical simulations of the Maxwell-Schrödinger equations without making the slowly varying amplitude approximation, and we obtained similar results.

\section{ELECTROMECHANICAL ANALOGY OF THE QASER}

Gain with no excited-state population produced by superradiant emission of radiation can be illustrated in a system of two pendulums weakly coupled by a spring, as shown in Fig. 3(a). Because of the weak coupling, mechanical energy flows back and forth between pendulums on a time scale much longer than the oscillation period of each pendulum. This process is analogous to photon absorption and reemission by atoms occurring with collective frequency $\Omega_{a}$. Thus, in the present analogy, the first pendulum corresponds to the XUV field, while the second one represents the atomic system.

One can obtain excitations of the atomic and radiation oscillators by periodical modulation of the coupling strength. Typically, such modulation also yields an unwanted periodic change in the oscillator frequency, which is analogous to the time-dependent Stark shift. One can avoid this shift in an arrangement in which the second pendulum is driven with a force $F=F_{0} \cos \left(\nu_{d} t\right) \phi_{1}$ proportional to the displacement of the first pendulum $\phi_{1}$. Such a force can be generated by the electrical feedback mechanism shown in Fig. 3(a), which yields the following equations of motion for the coupled pendulums (see Appendix C for details): (a)

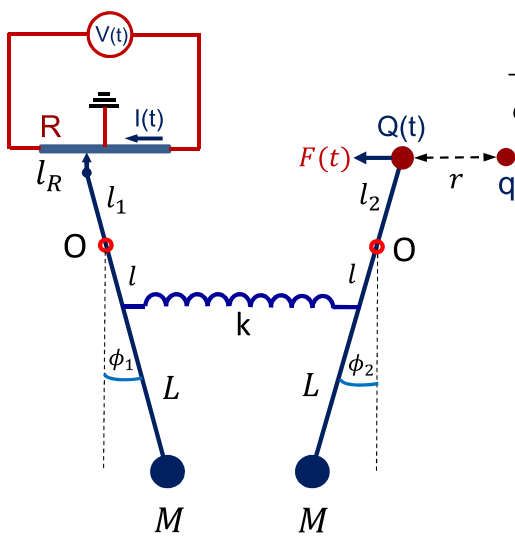

(b)

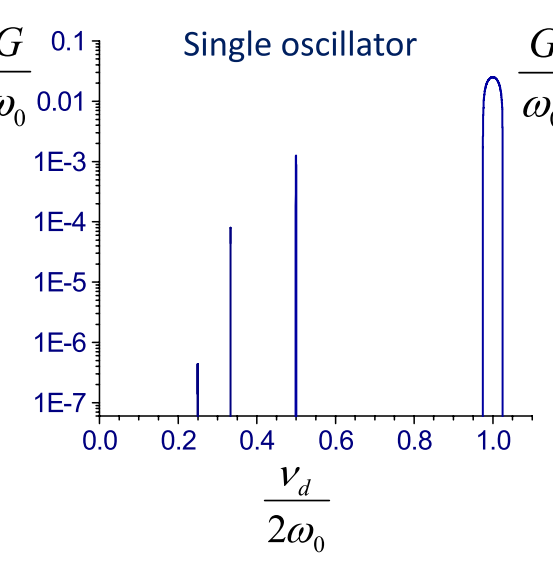

(c)

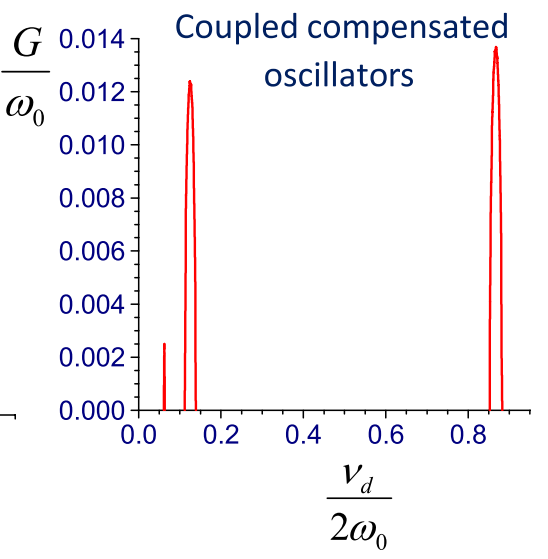

FIG. 3. (a) Electromechanical analog of the QASER. Masses $M$ are attached to conducting rods, which are pivoted at points $O$. The pendulums are weakly coupled with each other by a conducting spring. Pendulum 1 corresponds to the field and pendulum 2 to the atoms. A metallic sphere is attached to the top of the second pendulum, while the upper end of the first pendulum slides without friction along the resistor $R$ connected to the ac voltage supply $V(t)$. The middle point of the resistor is grounded. A charge $q$, placed at a fixed position, interacts with the charge on the metallic sphere $Q(t)$, which is proportional to $V(t)$ and to the displacement $\phi_{1}$ of the first pendulum from equilibrium. This interaction modulates the coupling strength between the pendulums. (b) Gain for a single oscillator as a function of modulation frequency $\nu_{d}$ obtained by numerical solution of Eq. (36) with $\Omega^{2} / \omega_{0}^{2}=0.25$ and $\delta=0.4$. The vertical axis has a logarithmic scale. (c) Gain for compensated coupled oscillators as a function of modulation frequency $\nu_{d}$ obtained by numerical solution of Eqs. (34) and (35) with $\Omega^{2} / \omega_{0}^{2}=0.25$ and $\delta=0.4$. 


$$
\begin{gathered}
\ddot{\phi}_{1}+\omega_{0}^{2} \phi_{1}-\Omega^{2} \phi_{2}=0, \\
\ddot{\phi}_{2}+\omega_{0}^{2} \phi_{2}-\Omega^{2}\left[1+\delta \cos \left(\nu_{d} t\right)\right] \phi_{1}=0,
\end{gathered}
$$

where $\omega_{0}$ is the frequency of the free pendulums, $\Omega \ll \omega_{0}$ is the coupling constant, and $\delta \ll 1$ is the modulation amplitude of the coupling strength.

Next, we compare gain for the single and coupled oscillators, assuming the same driving force for both systems. Namely, we assume that the two coupled pendulums are described by Eqs. (34) and (35), while evolution of the single oscillator obeys the Mathieu equation

$$
\ddot{\phi}+\left[\omega_{0}^{2}-\Omega^{2} \delta \cos \left(\nu_{d} t\right)\right] \phi=0 .
$$

In Figs. 3(b) and 3(c), we plot gain per unit time as a function of modulation frequency $\nu_{d}$ obtained by the numerical solution of Eq. (36) [Fig. 3(b)] and Eqs. (34) and (35) [Fig. 3(c)]. In simulations, we take $\Omega^{2} / \omega_{0}^{2}=0.25$ and $\delta=0.4$. For a single oscillator, the modulation yields exponential grow of the oscillation amplitude in the vicinity of parametric resonances $\nu_{d}=2 \omega_{0} / m$, where $m=1,2,3, \ldots$ However, gain for higher-order resonances (when $m>1$ ) becomes very small [see Fig. 3(b)]. In other words, if the driving frequency $\nu_{d}$ is much smaller than $\omega_{0}$, then excitation of a single oscillator is very inefficient. On the other hand, as is seen from Fig. 3(c), for coupled oscillators, there is strong resonance at the driving frequency

$$
\nu_{d}=\frac{\Omega^{2}}{\omega_{0}}=0.25 \omega_{0}
$$

At such a frequency, we see that gain $G=0.012 \omega_{0}$ is comparable to those for $\nu_{d} \approx 2 \omega_{0}$.

This simple mechanical example shows that a coupled system can be excited with high efficiency even if the driving frequency $\nu_{d}$ is much smaller than the frequency of the system's oscillations. Similarly, in the case of superradiant gain, the coupled field-atom system is efficiently excited by a low-frequency coherent drive, provided that we compensate for the deleterious time-dependent Stark shift.

\section{QASER PHYSICS FROM A GENERALIZED PERSPECTIVE}

The QASER is a device that generates high-frequency coherent radiation by driving an atomic ensemble with a much smaller frequency. The amplification mechanism of the QASER is governed by the difference combination parametric resonance that occurs when the driving-field frequency matches the frequency difference between two close high-frequency normal modes of the coupled lightatom system. The atoms interact with light collectively, which yields superradiant emission and reabsorption of the high-frequency radiation. This collective interaction determines the spacing between the system's normal modes and QASER gain.

The single oscillator described by Eq. (36) provides a simple example of parametric resonance. Such an oscillator has two natural frequencies (normal modes), $\pm \omega_{0}$. If we choose $\nu_{d}$ close to the difference between the natural frequencies, that is, $\nu_{d} \approx 2 \omega_{0}$, the oscillator phase-locks to the parametric variation and undergoes the first-order parametric resonance, absorbing energy at a rate proportional to the energy it already has.

Figure 3(b) shows that gain for the higher-order resonances occurring at lower frequencies, $\nu_{d} \approx 2 \omega_{0} / \mathrm{m}, \mathrm{m}=$ $2,3, \ldots$, is very small, and thus, excitation of the system by the higher-order resonances is inefficient. Nevertheless, as we show, it is possible to effectively excite high-frequency oscillations with a low-frequency drive if the system has a pair of high-frequency normal modes with small spacing. Modulation of parameters of such a system at a small frequency equal to the spacing between the two close normal modes can display the first-order parametric resonance and, thus, yield an efficient excitation of the high-frequency oscillations.

Two weakly coupled harmonic oscillators, described by Eqs. (34) and (35) with $\Omega \ll \omega_{0}$, give an example of such a system. This system has a pair of close normalmode frequencies, $\omega_{ \pm}=\sqrt{\omega_{0}^{2} \pm \Omega^{2}} \approx \omega_{0} \pm \Omega^{2} / 2 \omega_{0}$. If the modulation frequency matches the frequency difference between those modes, namely, $\nu_{d}=\omega_{+}-\omega_{-} \approx$ $\Omega^{2} / \omega_{0}$, the system displays resonance with high gain [see Eq. (C10)],

$$
G=\frac{\delta \cdot \nu_{d}}{8},
$$

which is proportional to $\delta$, rather than $\delta^{m}(m>1)$, which would be the case for the higher-order resonances. This result is illustrated in Fig. 3(c).

The QASER operates by the same principle. Indeed, propagation of light [having Rabi frequency $\Omega(t, \mathbf{r})$ ] through the medium of two-level atoms is described by the coupled Maxwell-Schrödinger equations, which, without making the slowly varying amplitude approximation, read

$$
\begin{aligned}
& \left(\frac{\partial^{2}}{\partial t^{2}}-c^{2} \nabla^{2}\right) \Omega(t, \mathbf{r})=-2 \frac{\Omega_{a}^{2}}{\omega_{a b}} \frac{\partial^{2} \rho_{a b}}{\partial t^{2}}, \\
& \frac{\partial \rho_{a b}}{\partial t}+i \omega_{a b} \rho_{a b}=i \Omega(t, \mathbf{r})\left(\rho_{b b}-\rho_{a a}\right) .
\end{aligned}
$$

Equations (39) and (40) describe two coupled oscillators. To find the normal modes of the coupled system for a weak atomic excitation, one can look for the solution of Eqs. (39) and (40) in the form $\Omega, \rho_{a b} \propto \exp (i k z-i \nu t)$, which, for weak coupling and a mode with the wave number $k$ near $\omega_{a b} / c$, yields two close frequencies, 


$$
\nu_{ \pm}=\frac{1}{2}\left[\omega_{a b}+c k \pm \sqrt{\left(\omega_{a b}-c k\right)^{2}+4 \Omega_{a}^{2}}\right],
$$

where we assume that initially the atoms are in the ground state, that is, $\rho_{b b}-\rho_{a a} \approx 1$. Thus, if we modulate the system's parameters at the small frequency

$$
\nu_{d}=\nu_{+}-\nu_{-}=\sqrt{\left(\omega_{a b}-c k\right)^{2}+4 \Omega_{a}^{2}} \ll \nu_{ \pm},
$$

the high-frequency modes $\nu_{ \pm}$can be excited with large gain via the first-order parametric resonance. Since in the present problem the wave number $k$ is a continuous parameter, one can satisfy the resonance condition for $\nu_{d} \geq 2 \Omega_{a}$. Namely, for such $\nu_{d}$, there are always two normal modes (value of $k$ ) that are in resonance with the driving field.

Terms in the right-hand side of Eqs. (39) and (40) describe the coupling between two oscillators. Nonlinearity of the coupling allows us to modulate the coupling strength by driving atoms with a low-frequency field, which serves as an energy source for the QASER operation. Such driving, however, also produces an unwanted ac Stark shift of the atomic transition, which must be compensated in order to achieve gain.

The amplification mechanism of the QASER, namely, the difference combination parametric resonance between two close normal modes, can appear in various physical systems. In principle, such a mechanism can be used to generate coherent gamma radiation in nuclear ensembles and to control propagation of gamma rays through crystals on a picosecond time scale; we will discuss this possibility elsewhere [25].

The study of variable-parameter (or parametric) processes in electronic circuits in the 1950s led to the discovery of a frequency-conversion mechanism in which the energy is fed from the source that modulates the circuit parameters at frequency $\nu_{d}$ to two circuit-normal modes of lower frequencies, $\omega_{1}$ and $\omega_{2}$, obeying the relation $[26,27]$

$$
\nu_{d}=\omega_{1}+\omega_{2}
$$

This yields amplification of frequencies $\omega_{1}$ and $\omega_{2}$. A variable inductor or a capacitor suitably coupled to two resonance circuits is an example of such a parametric amplifier [26]. A few years later, the principle of parametric amplification was proposed for generation of light waves at frequencies $\omega_{1}$ and $\omega_{2}$ in nonlinear optical crystals [28-30]. The first successful attainment of parametric oscillation at optical frequencies was reported in 1965 in $\mathrm{LiNbO}_{3}$ [31]. The progress in parametric amplification and oscillation has been the subject of many review papers (see, e.g., [32-34]). Nowadays, the optical parametric oscillators (OPOs) are commercially available.

An OPO converts an input laser wave with frequency $\nu_{d}$ into two output waves of lower frequency satisfying Eq. (43) by means of the second-order nonlinear optical interaction in crystal. The phase matching plays a decisive role here. However, QASER operation does not require phase matching, and in contrast to an OPO, it generates light at frequencies obeying the relation

$$
\nu_{d}=\omega_{2}-\omega_{1} .
$$

It seems that the study of the electronic circuits back in the 1950s largely missed the possibility of generating such frequencies. This omission is not surprising because the QASER mechanism requires compensation of the ac frequency shift (Stark shift) and asymmetric modulation of the coupling between oscillators [see Eqs. (34) and (35)], which is usually not the case.

Resonances described by Eqs. (43) and (44) are known in applied mechanics as combination resonances. They affect the dynamic stability of structures and appear in systems having multiple degrees of freedom. The literature on combination resonances in such systems is abundant (see, e.g., [35-37]). In particular, the sum (43) and the difference (44) combination resonances have been theoretically studied in connection with the parametric instability of a cantilevered column under periodic loads [38] and shear-deformable laminated plates [39]. It has also been suggested that the failure of the high-pressure compressor of jet engines (which was occurring in the past) can be due to the difference combination resonance when the rotating speed of the rotor matches the difference between natural frequencies of the rotating and static seals [40].

One should mention that combination resonances can occur only under certain conditions. Small oscillations in modulated systems with many degrees of freedom can be described by coupled differential equations, which in matrix notation read

$$
\ddot{q}+C \dot{q}+\left[A+B \cos \left(\nu_{d} t\right)\right] q=0,
$$

where $q$ is a vector of generalized coordinates, while $A, B$, and $C$ are matrices. $A$ is a diagonal matrix, while matrix $C$ denotes gyroscopic terms and is usually antisymmetric. It has been shown that if $C=0$ and $B$ is a symmetric matrix (as is the case in many applications), then the difference combination resonance does not occur. If $B$ is symmetric, then Eq. (45) can be derived from a potential function, and hence, terms $B q$ are conservative forces. In such Hamiltonian systems, only the sum combination resonances (43) can be excited [41,42], which is the reason why the sum combination resonance is a frequent phenomenon.

In other words, if the difference combination resonance is possible in systems with no gyroscopic terms, then the system must be nonconservative [43]. This is the case for the electromechanical analog of the QASER shown in Fig. 3(a), which possesses dissipation in resistor $R$. The results are changed as soon as gyroscopic terms are involved [41]. Now, the difference parametric resonance can occur even if $B$ is symmetric. Hamiltonian systems with gyroscopic forces (e.g., the Lorentz magnetic force) can 
have both the sum and the difference combination resonances.

While there is plenty of literature on combination resonances, there do not seem to be any experimental examples of difference resonances [43]. In fact, such a device might be somewhat strange. If we had $\omega_{1} \approx \omega_{2}$, then $\nu_{d}=\omega_{2}-\omega_{1}$ would be small, perhaps several orders of magnitude smaller. So, a difference combination resonance would generate a high frequency from a low-frequency excitation.

In the next section, we present an electronic circuit experiment that has just such a property. The experiment can be easily described theoretically in a somewhat ideal electronic circuit model, shown in Fig. 4. The model consists of two ideal LC circuits that are weakly coupled by an inductor $L_{0}$ and a multiplier $M$. The output voltage $V_{\text {out }}$ of the multiplier is proportional to the product of the two input voltages $V(t)$ and $V_{1}: V_{\text {out }}=\kappa V(t) V_{1}$, where $\kappa$ is the gain of the multiplier and $V(t)=V_{0} \cos \left(\nu_{d} t\right)$ is the voltage produced by a function generator. The multiplier makes coupling between the two LC circuits nonreciprocal; in other words, matrix $B$ in Eq. (45) is not symmetric for such a system. The multiplier has high input resistance, so one can disregard its input current.

Let the electric charges of the capacitors be $Q_{1}$ and $Q_{2}$. Applying Kirchhoff's laws to the system, we obtain (for notation, see Fig. 4)

$$
\begin{gathered}
L_{1}\left(\ddot{Q}_{1}+\dot{I}_{0}\right)+\frac{Q_{1}}{C_{1}}=0, \\
L_{2}\left(\ddot{Q}_{2}-\dot{I}_{0}\right)+\frac{Q_{2}}{C_{2}}-\kappa \frac{Q_{1}}{C_{1}} V(t)=0,
\end{gathered}
$$

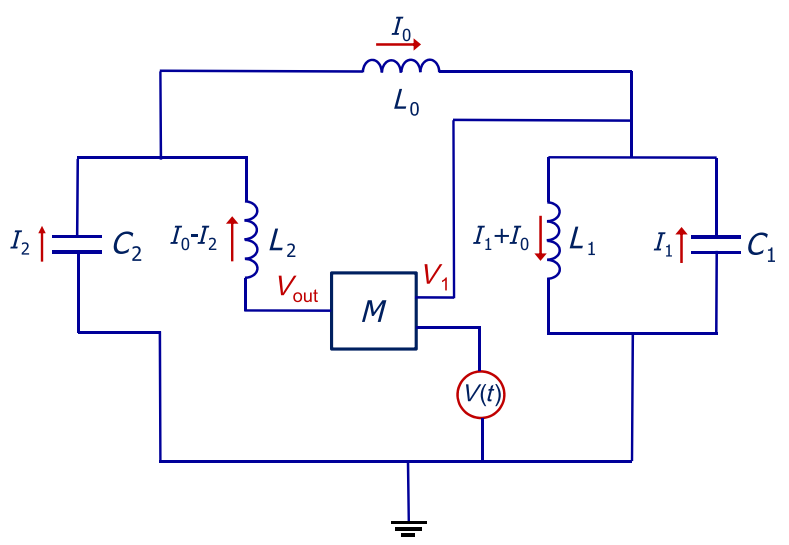

FIG. 4. Electronic circuit analog of the QASER. Two LC circuits weakly coupled by an inductor $L_{0}$ correspond to the atoms and to the field. Modulation of the coupling strength is provided by a feedback mechanism in which voltage $V_{1}$ from the capacitor $C_{1}$ is applied to the input terminal of the multiplier $\mathrm{M}$; its output voltage $V_{\text {out }}$ is proportional to the product of the two input voltages $V(t)$ and $V_{1}$, where $V(t)=V_{0} \cos \left(\nu_{d} t\right)$ is the voltage produced by a function generator.

$$
L_{0} \dot{I}_{0}=\frac{Q_{1}}{C_{1}}-\frac{Q_{2}}{C_{2}} .
$$

Elimination of $\dot{I}_{0}$ from Eqs. (46)-(48) yields two coupled equations,

$$
\begin{gathered}
\ddot{Q}_{1}+\frac{Q_{1}}{L_{1} C_{1}}\left(1+\frac{L_{1}}{L_{0}}\right)-\frac{Q_{2}}{L_{0} C_{2}}=0, \\
\ddot{Q}_{2}+\frac{Q_{2}}{L_{2} C_{2}}\left(1+\frac{L_{2}}{L_{0}}\right)-\frac{Q_{1}}{L_{0} C_{1}}\left[1+\kappa \frac{L_{0}}{L_{2}} V(t)\right]=0 .
\end{gathered}
$$

Assuming that $L_{1}=L_{2}=L, C_{1}=C_{2}=C$ and introducing the notations

$$
\begin{gathered}
\omega_{0}^{2}=\frac{1}{L C}\left(1+\frac{L}{L_{0}}\right), \\
\Omega^{2}=\frac{1}{L_{0} C}, \quad \delta=\kappa V_{0} \frac{L_{0}}{L},
\end{gathered}
$$

one can write Eqs. (49) and (50) as

$$
\begin{gathered}
\ddot{Q}_{1}+\omega_{0}^{2} Q_{1}-\Omega^{2} Q_{2}=0, \\
\ddot{Q}_{2}+\omega_{0}^{2} Q_{2}-\Omega^{2}\left[1+\delta \cos \left(\nu_{d} t\right)\right] Q_{1}=0,
\end{gathered}
$$

which are identical to Eqs. (34) and (35) of the previous section; thus, the present electronic circuit model displays similar parametric excitation.

The two LC circuits are weakly coupled, provided that $L_{0} \gg L$. Driving the system with small frequency $\nu_{d} \approx$ $\Omega^{2} / \omega_{0}=\omega_{0} L / L_{0} \ll \omega_{0}$ yields the difference resonance that results in the efficient excitation of oscillations in the $\mathrm{LC}$ circuits at the high natural frequencies $\omega_{0}\left(1 \pm L / 2 L_{0}\right)$. In Appendix E, we perform a computer experiment and demonstrate in detail how the high-frequency oscillations are generated in a realistic electronic circuit model that includes resistance and internal capacitance of the inductors. In numerical simulations, we choose parameters of the circuit elements that are readily available in an undergraduate electronics laboratory.

\section{EXPERIMENTAL DEMONSTRATION OF THE QASER AMPLIFICATION MECHANISM IN AN ELECTRONIC CIRCUIT}

In this section, we present an experiment that illustrates the QASER amplification mechanism in an electronic system, as shown in Fig. 5. The system consists of two RLC circuits weakly coupled by a capacitor and a multiplier $M$ that makes coupling nonreciprocal. In our experiment, $M$ is an AD633JN analog multiplier connected as a linear amplitude modulator. Its output voltage $V_{\text {out }}$ is given by

$$
V_{\text {out }}=\frac{V_{1} \cdot V(t)}{10 \mathrm{~V}}+V(t),
$$




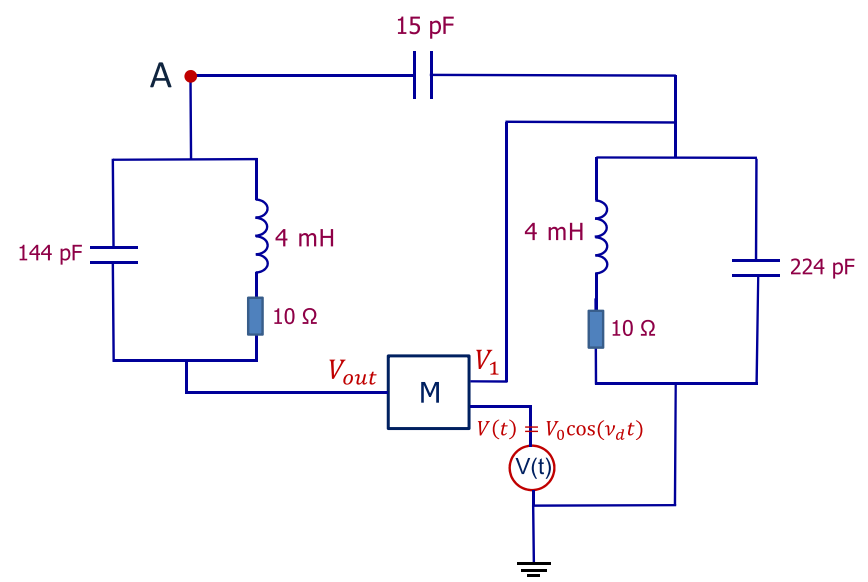

FIG. 5. Experimental setup: Two RLC circuits are connected by a capacitor and a multiplier $M$, which yields nonreciprocal coupling between the two circuits. The transfer function of the multiplier is given by Eq. (55). Sinusoidal voltage from a function generator is applied to one of the multiplier's inputs, which produces modulation of the coupling strength. Voltage $V_{A}(t)$ at the point $A$ is measured by an oscilloscope.

where $V(t)$ is the voltage produced by the DS345 synthesized function generator, which is fed to the carrier input of $\mathrm{AD} 633 \mathrm{JN}$, and $V_{1}$ is the voltage applied to the modulation input (see Fig. 5). The circuit is set up on a breadboard using an apparatus that is available in an undergraduate electronics laboratory. The circuit parameters are indicated in Fig. 5. The breadboard has self-capacitance of a few $\mathrm{pF}$.

The circuit parameters are chosen such that the system's natural frequencies are close to each other. To compensate for the capacitance of the multiplier, we designed the
RLC circuits with different $C$ 's. We measured the natural frequencies of the system to be $\omega_{1}=196 \mathrm{kHz}$ and $\omega_{2}=222 \mathrm{kHz}$. Thus, the frequency difference between normal modes is $\Delta \omega=26 \mathrm{kHz}$. The quality factor of the circuit is $Q=\sqrt{L / C} / R \approx 400$, which yields the resonance bandwidth $0.5 \mathrm{kHz}$.

In our experiment, we modulate the coupling between the RLC circuits by applying sinusoidal voltage of frequency $\nu_{d}$ and amplitude $V_{0}$,

$$
V(t)=V_{0} \cos \left(\nu_{d} t\right)
$$

to the multiplier input and measure the voltage $V_{A}(t)$ at the point $A$ of the circuit (see Fig. 5) using the Tektronix TDS 210 oscilloscope. We examine how $V_{A}(t)$ changes under variation of $\nu_{d}$ and $V_{0}$.

We find that when $\nu_{d}=\Delta \omega=26 \mathrm{kHz}$ and $V_{0}$ is greater than a threshold value, the system starts to oscillate at high frequencies. The measured spectrum of such oscillations is given in Fig. 6(a) for $V_{0}=0.9 \mathrm{~V}$ (solid lines). The spectrum has a peak at the low driving frequency $\nu_{d}=26 \mathrm{kHz}$ and several high-frequency components at $\omega=\omega_{1,2}$, plus two sidebands at $\omega_{1}-\nu_{d}=$ $170 \mathrm{kHz}$ and $\omega_{2}+\nu_{d}=248 \mathrm{kHz}$. In the figure, vertical dashed lines indicate the position of higher harmonics of the driving frequency. Clearly, the observed highfrequency oscillations are not produced by the higher harmonics of $\nu_{d}$, which do not overlap with the generated spectral components.

Amplification of the high-frequency oscillations occurs when parametric gain (which depends on $V_{0}$ ) exceeds losses. To demonstrate such a threshold behavior, we measured the system's oscillation amplitude at the natural frequency $\omega_{2}=222 \mathrm{kHz}$ as a function of $V_{0}$ for (a)

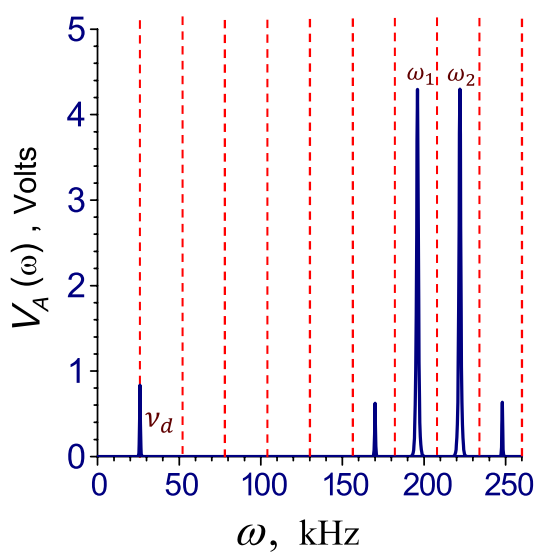

(b)

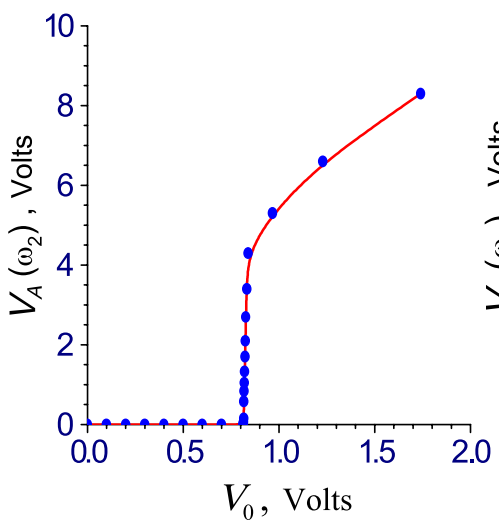

(c)

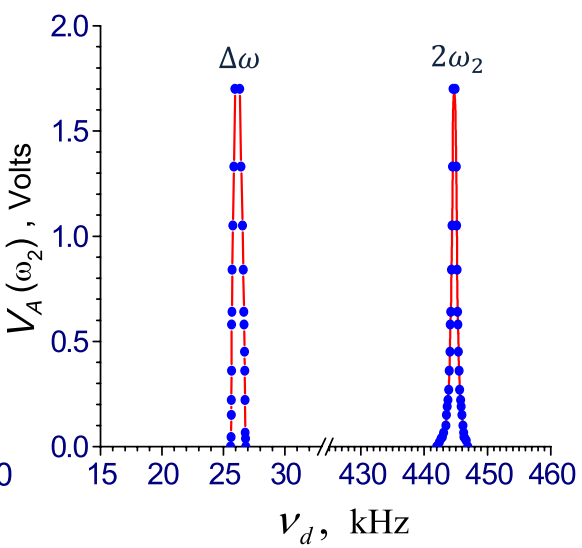

FIG. 6. (a) Measured spectrum of the system's oscillations for driving frequency $\nu_{d}=26 \mathrm{kHz}$ and driving amplitude $V_{0}=0.9 \mathrm{~V}$ (solid lines). Vertical dashed lines at $\omega=m \nu_{d}, m=1,2, \ldots$, indicate the position of higher harmonics of the driving frequency. (b) Dependence of the system's oscillation amplitude at the natural frequency $\omega_{2}=222 \mathrm{kH} z$ on $V_{0}$ for $\nu_{d}=26 \mathrm{kH} z$. (c) Dependence of the system's oscillation amplitude at the natural frequency $\omega_{2}=222 \mathrm{kHz}$ on $\nu_{d}$ for $V_{0}=0.825 \mathrm{~V}$. In all plots, the voltage is measured at the point $A$ (see Fig. 5). 
$\nu_{d}=26 \mathrm{kHz}$. The results are shown in Fig. 6(b) (dots). For $V_{0}<0.814 \mathrm{~V}$, there is no amplification at any frequency, while for $V_{0} \geq 0.814 \mathrm{~V}$, the net gain becomes positive and high frequencies are efficiently generated by the low-frequency drive.

Finally, to illustrate the resonant nature of the amplification mechanism, we measured the system's oscillation amplitude at the natural frequency $\omega_{2}=222 \mathrm{kHz}$ as a function of $\nu_{d}$ for $V_{0}=0.825 \mathrm{~V}$ [see Fig. 6(c)]. We found that high-frequency generation occurs only when $\nu_{d}$ is near $\Delta \omega=26 \mathrm{kHz}$ or $2 \omega_{2}=444 \mathrm{kHz}$. This result agrees with our theoretical findings displayed in Fig. 3(c).

To the best of our knowledge, the present results provide the first experimental demonstration of difference combination resonance, that is, where the system generates oscillations at high natural frequencies $\omega_{1}$ and $\omega_{2}$ when forced with a low frequency $\nu_{d}=\omega_{2}-\omega_{1}$. Such a mechanism holds promise for generating high-frequency electromagnetic oscillations in electronic circuits (e.g., developing $\mathrm{THz}$ generators) and highfrequency (XUV or x-ray) coherent light by means of a low-frequency drive.

\section{SUMMARY AND OUTLOOK}

We have found a new way to obtain quantum gain with no population in the excited state by means of quantum amplification by superradiant emission of radiation (QASER). In our approach, light amplification occurs because of the difference combination parametric resonance between collective oscillations of the coupled light-atom system and the external (e.g., infrared) driving field, which can yield exponential growth of the seed pulse at high (e.g., XUV) atomic frequency. To achieve gain, one must suppress the unwanted time-dependent Stark shift of the atomic transition energy, which can be realized in various schemes (see Appendix F for details on the ac Stark shift compensation in many-level atoms).

The QASER mechanism is different from the LASER (which requires nonzero excited-state population) and the well-known multiphoton resonant excitation (which is a single-atom phenomenon rather than a collective effect). In particular, excited-state population grows with time differently for the multiphoton excitation and the QASER, as indicated in Eqs. (20) and (21). We also show that the QASER is analogous to excitation of a system of two weakly coupled oscillators, which displays the difference combination resonance when the low modulation frequency matches the frequency difference between two close normal modes of the coupled system.

We carried out an experiment that demonstrates the QASER amplification mechanism in an electronic circuit. To the best of our knowledge, the present experiment is the first experimental demonstration of the difference combination resonance that yields excitation of high-frequency oscillations by a low-frequency drive.
The QASER is different from the OPO in several ways. The OPO requires phase matching, but the QASER does not. QASER emission is backward relative to the drive, while in the OPO, it is forward. The ac Stark shift compensation is key to the QASER operation, but not to the OPO. The QASER can operate in a gas, but the OPO requires a noncentrosymmetric nonlinear crystal. Finally, the OPO is a sum-frequency device; namely, frequencies $\omega_{1}$ and $\omega_{2}$ produced by the OPO from the driving field $\nu_{d}$ obey the relation $\nu_{d}=\omega_{1}+$ $\omega_{2}$. In other words, the OPO generates lower frequencies than $\nu_{d}$. The QASER is the difference frequency device for which $\nu_{d}=\omega_{2}-\omega_{1}$. More precisely, the QASER can generate higher frequencies by means of superradiant resonance.

Such a superradiant resonance holds promise for development of a new class of radiation sources that generate high-frequency (e.g., XUV or x-ray) coherent light by utilizing a low-frequency (e.g., infrared) coherent source. However, as is always the case in research, the present scheme surely involves many challenges, open questions, and unknowns. For example, what about all the other level pairs? Will the device tend to oscillate on all of these pairs? We think not. One can select a narrow window of atomic frequencies, e.g., by sending an XUV seed pulse, or compensate the ac Stark shift only for a particular atomic transition. This will be discussed further in forthcoming papers.

Will ionization of the excited states by the intense driving field be a problem? No. One can choose the driving field to be weak enough so that we will not have excessive multiphoton ionization. This result is possible since the present collective resonant excitation is a much stronger effect than the usual multiphoton mechanism. Can we operate more efficiently at high pressures and/or with shorter wavelengths of the driving field, etc.? This is an open question. There are surely many such open questions that need to be investigated. In Appendix F, we discuss possible experiments that could demonstrate QASER operation in a noble gas (e.g., $\mathrm{He}$ ) or a gas of alkali atoms (e.g., $\mathrm{Rb}$ or $\mathrm{Na}$ ) by using available laser technology.

The intent of the present paper is to stimulate interest in this new approach to the generation of short-wavelength coherent light. If we accomplish that goal, this paper will have served its purpose.

\section{ACKNOWLEDGMENTS}

We thank A. Sokolov, A. Belyanin, S. Harris, O. Kocharovskaya, V. Kocharovsky, Yu. Rostovtsev, W. Schleich, G. Welch, and X. Zhang for useful discussions. We gratefully acknowledge support of the National Science Foundation Grants No. PHY-1241032 and No. PHY-1205868, and the Robert A. Welch Foundation (Grant No. A-1261). 


\section{APPENDIX A: EVOLUTION EQUATION FOR SUPERRADIANT PULSE}

Under the influence of the electromagnetic field $\Omega$, atomic population and coherence $\rho_{a b}$ evolve according to the equations

$$
\begin{gathered}
\dot{\rho}_{a a}=-\gamma \rho_{a a}-i\left(\Omega^{*} \rho_{a b}-\text { c.c. }\right) \\
\rho_{a a}+\rho_{b b}=1, \\
\dot{\rho}_{a b}=-\left(i \omega_{a b}+\gamma_{\mathrm{tot}}\right) \rho_{a b}+i \Omega\left(\rho_{b b}-\rho_{a a}\right) .
\end{gathered}
$$

We consider the regime of superradiance. Thus, one can omit spontaneous decay $\gamma$ and decoherence $\gamma_{\text {tot }}$, provided that their rates are small compared to gain per unit time, $G$.

Here, we are interested in the generation of coherent radiation at atomic frequency $\omega_{a b}$, and we study the evolution of a weak superradiant pulse $\Omega_{s}$ with carrier frequency $\omega_{a b}$ propagating along the $z$ axis through the medium. In Eqs. (A1)-(A3), one can write the total field, the total coherence, and population of the excited state as

$$
\begin{aligned}
& \Omega=\Omega^{\text {drive }}+\Omega^{\text {super }}, \\
& \rho_{a b}=\rho_{a b}^{\text {drive }}+\rho_{a b}^{\text {super }}, \\
& \rho_{a a}=\rho_{a a}^{\text {drive }}+\rho_{a a}^{\text {super }},
\end{aligned}
$$

where $\Omega^{\text {super }}, \rho_{a b}^{\text {super }}$, and $\rho_{a a}^{\text {super }}$ are small, fast-oscillating corrections that, in the slowly varying envelope approximation, have the form

$$
\begin{gathered}
\Omega^{\text {super }}=\Omega_{s}(t, z) e^{-i \omega_{a b} t+i \omega_{a b} z / c}+\text { c.c., } \\
\rho_{a b}^{\text {super }}=\rho_{a b}^{s}(t, z) e^{-i \omega_{a b} t+i \omega_{a b} z / c}, \\
\rho_{a a}^{\text {super }}=\rho_{a a}^{s}(t, z) e^{-i \omega_{a b} t+i \omega_{a b} z / c}+\text { c.c. }
\end{gathered}
$$

Here, $\Omega_{s}(t, z), \rho_{a b}^{s}(t, z)$, and $\rho_{a a}^{s}(t, z)$ are slowly varying functions as compared to the fast-oscillating exponentials. Please note that $\Omega^{\text {drive }}$ can be complex, while we choose modes of the field $\Omega^{\text {super }}$ to be real functions.

Plugging Eqs. (A4)-(A9) into Eqs. (A1)-(A3) and neglecting higher harmonics, we obtain the following equations for $\Omega_{s}(t, z), \rho_{a b}^{s}(t, z)$, and $\rho_{a a}^{s}(t, z)$ :

$$
\begin{gathered}
\dot{\rho}_{a b}^{s}=i \Omega_{s}\left(1-2 \rho_{a a}^{\mathrm{drive}}\right)-2 i \Omega^{\mathrm{drive}} \rho_{a a}^{s}, \\
i \dot{\rho}_{a a}^{s}+\omega_{a b} \rho_{a a}^{s}=\Omega_{s}\left(\rho_{a b}^{\mathrm{drive}}-\text { c.c. }\right)+\Omega^{\mathrm{drive}{ }^{*}} \rho_{a b}^{s} .
\end{gathered}
$$

Since the emission field is weak (the regime of linear gain), it does not affect the evolution of $\rho_{a b}^{\text {drive }}$ and $\rho_{a a}^{\text {drive }}$, which are governed by separate equations:

$$
\begin{gathered}
\dot{\rho}_{a b}^{\text {drive }}=-i \omega_{a b} \rho_{a b}^{\text {drive }}+i \Omega^{\text {drive }}\left(1-2 \rho_{a a}^{\mathrm{drive}}\right), \\
\dot{\rho}_{a a}^{\text {drive }}=i \Omega^{\text {drive }} \rho_{b a}^{\text {drive }}-i \Omega^{\text {drive* }} \rho_{a b}^{\text {drive }} .
\end{gathered}
$$

Equations (A10)-(A13) should be supplemented by the Maxwell equation for the superradiant pulse, which in the slowly varying envelope approximation reads

$$
\left(\frac{\partial}{\partial t}+c \frac{\partial}{\partial z}\right) \Omega_{s}=i \Omega_{a}^{2} \rho_{a b}^{s} .
$$

In Eq. (A11), the term $i \dot{\rho}_{a a}^{s}$ can be treated as a small perturbation. Then, Eq. (A11) gives

$$
\begin{aligned}
\rho_{a a}^{s} \approx & 2 i \operatorname{Im}\left[\rho_{a b}^{\mathrm{drive}}\right] \frac{\Omega_{s}}{\omega_{a b}}+\frac{\Omega^{\mathrm{drive}^{*}}}{\omega_{a b}} \rho_{a b}^{s} \\
& -\frac{i}{\omega_{a b}^{2}} \frac{d}{d t}\left(2 i \operatorname{Im}\left[\rho_{a b}^{\mathrm{drive}}\right] \Omega_{s}+\Omega^{\mathrm{drive}^{*}} \rho_{a b}^{s}\right) .
\end{aligned}
$$

In this equation, we keep terms up to the order $1 / \omega_{a b}^{2}$. Since $\operatorname{Im}\left[\rho_{a b}^{\text {drive }}\right] \propto 1 / \omega_{a b}$, Eq. (A15) reduces to

$$
\begin{aligned}
\rho_{a a}^{s} \approx & 2 i \operatorname{Im}\left[\rho_{a b}^{\mathrm{drive}}\right] \frac{\Omega_{s}}{\omega_{a b}}+\frac{\Omega^{\mathrm{drive}^{*}}}{\omega_{a b}} \rho_{a b}^{s} \\
& -\frac{i}{\omega_{a b}^{2}} \frac{d}{d t}\left(\Omega^{\mathrm{drive}^{*}} \rho_{a b}^{s}\right) .
\end{aligned}
$$

Plugging Eq. (A16) into Eq. (A10) yields

$$
\begin{aligned}
(1 & \left.+\frac{2}{\omega_{a b}^{2}}\left|\Omega^{\text {drive }}\right|^{2}\right) \dot{\rho}_{a b}^{s} \\
= & -2\left(\frac{i}{\omega_{a b}}\left|\Omega^{\text {drive }}\right|^{2}+\frac{\Omega^{\text {drive }} \dot{\Omega}^{\text {drive }}}{\omega_{a b}^{2}}\right) \rho_{a b}^{s} \\
& +i\left(1-2 \rho_{a a}^{\text {drive }}-i \frac{4 \Omega^{\text {drive }}}{\omega_{a b}} \operatorname{Im}\left[\rho_{a b}^{\text {drive }}\right]\right) \Omega_{s} .
\end{aligned}
$$

Taking the time derivative from both sides of Eq. (A14), using Eq. (A17), and keeping the leading-order terms, we find the following evolution equation for the superradiant pulse:

$$
\begin{aligned}
& \left(\frac{\partial}{\partial t}+i \Delta \omega_{a b}(t)+\frac{2 \Omega^{\text {drive }} \dot{\Omega}^{\text {drive }^{*}}}{\omega_{a b}^{2}}\right)\left(c \frac{\partial}{\partial z}+\frac{\partial}{\partial t}\right) \Omega_{s} \\
& \quad+\Omega_{a}^{2}\left(1-2 \rho_{a a}^{\text {drive }}-\frac{2\left|\Omega^{\text {drive }}\right|^{2}}{\omega_{a b}^{2}}-i \frac{4 \Omega^{\text {drive }}}{\omega_{a b}} \operatorname{Im}\left[\rho_{a b}^{\text {drive }}\right]\right) \Omega_{s} \\
& =0,
\end{aligned}
$$

where

$$
\Delta \omega_{a b}(t)=\frac{2\left|\Omega^{\text {drive }}(t, z)\right|^{2}}{\omega_{a b}}
$$

is the time-dependent Stark shift of the atomic transition frequency that is proportional to the instantaneous intensity of the driving field. 
APPENDIX B: GAIN PRODUCED BY COLLECTIVE PARAMETRIC RESONANCE

In order to achieve gain with no population in the excited state, one should suppress the time-dependent Stark shift $\Delta \omega_{a b}(t)$ that washes out the parametric resonance. This suppression can be accomplished in several ways, which we discuss in the present section.

\section{Nearly circularly polarized driving field}

The time-dependent Stark shift can be compensated if the driving-field Rabi frequency has the form

$$
\Omega^{\text {drive }}(t, z)=\Omega_{1} e^{i \nu_{d} t-i k_{d} z}+\Omega_{2} e^{-i \nu_{d} t+i k_{d} z},
$$

where $\Omega_{1}$ and $\Omega_{2}$ are real constants and $k_{d}$ is the wave number of the driving field. As we show below, for a certain ratio of $\Omega_{2} / \Omega_{1}$, the Stark shift vanishes. Such a situation can be realized for an elliptically polarized driving field $\mathbf{E}^{\text {drive }}$ if the dipole matrix element of the two-level atom is

$$
\mathbf{d}_{a b}=d_{x} \hat{x}+i d_{y} \hat{y},
$$

where $d_{x}$ and $d_{y}$ are real. This is, e.g., the case if the excited state $a$ corresponds to a $p_{x}+i p_{y}$ atomic orbital and $b$ is an $s$ state.

We assume that $\left|\Omega^{\text {drive }}\right|, \nu_{d} \ll \omega_{a b}$, that is, the driving field produces small modulations. Then, Eqs. (A12) and (A13) yield that under the influence of the driving field (which is turned on adiabatically), atomic coherence and population evolve as

$$
\begin{array}{r}
\rho_{a b}^{\mathrm{drive}}=\frac{\Omega_{1}}{\omega_{a b}+\nu_{d}} e^{i\left(\nu_{d} t-k_{d} z\right)}+\frac{\Omega_{2}}{\omega_{a b}-\nu_{d}} e^{-i\left(\nu_{d} t-k_{d} z\right)}, \\
\rho_{a a}^{\mathrm{drive}} \approx \\
\frac{\Omega_{1}^{2}}{\left(\omega_{a b}+\nu_{d}\right)^{2}}+\frac{\Omega_{2}^{2}}{\left(\omega_{a b}-\nu_{d}\right)^{2}} \\
+2 \frac{\Omega_{1} \Omega_{2}}{\omega_{a b}^{2}-\nu_{d}^{2}} \cos \left(2 \nu_{d} t-2 k_{d} z\right) .
\end{array}
$$

Coherence given by Eq. (B3) oscillates with the frequency of the driving field $\nu_{d} \ll \omega_{a b}$, and hence, it does not generate light at the atomic frequency $\omega_{a b}$.

Taking into account Eqs. (B1) and (B3), we obtain

$$
\begin{gathered}
\left|\Omega^{\text {drive }}\right|^{2}=\Omega_{1}^{2}+\Omega_{2}^{2}+2 \Omega_{1} \Omega_{2} \cos \left(2 \nu_{d} t-2 k_{d} z\right), \quad(\text { B5) } \\
\Omega^{\text {drive }} \dot{\Omega}^{\text {drive* }}=i \nu_{d}\left(\Omega_{2}^{2}-\Omega_{1}^{2}\right)-2 \nu_{d} \Omega_{1} \Omega_{2} \sin \left(2 \nu_{d} t-2 k_{d} z\right),
\end{gathered}
$$

$$
\operatorname{Im}\left[\rho_{a b}^{\mathrm{drive}}\right]=\left(\frac{\Omega_{1}}{\omega_{a b}+\nu_{d}}-\frac{\Omega_{2}}{\omega_{a b}-\nu_{d}}\right) \sin \left(\nu_{d} t-k_{d} z\right) .
$$

Assuming that $\Omega_{2} \ll \Omega_{1}$ (a nearly circularly polarized field), Eq. (A18) can be written as

$$
\begin{aligned}
\left(\frac{\partial}{\partial t}\right. & \left.+\frac{2 i \Omega_{1}^{2}}{\omega_{a b}}+\frac{4 i \Omega_{1} \Omega_{2}}{\omega_{a b}} \cos \left(2 \nu_{d} t-2 k_{d} z\right)\right)\left(c \frac{\partial}{\partial z}+\frac{\partial}{\partial t}\right) \Omega_{s} \\
& +\Omega_{a}^{2}\left(1-\frac{2 \Omega_{1}^{2}}{\omega_{a b}^{2}}-\frac{2 \Omega_{1}^{2}}{\omega_{a b}^{2}} e^{2 i \nu_{d} t-2 i k_{d} z}\right) \Omega_{s}=0 .
\end{aligned}
$$

To obtain quick insight on the gain conditions, we consider a spatially uniform driving field $\left(k_{d}=0\right)$ and look for solutions of Eq. (B8) in the form

$$
\Omega_{s}(t, z)=\Omega_{s}(t),
$$

which yields the following equation for $\Omega_{s}(t)$ :

$$
\begin{aligned}
\left(\frac{\partial}{\partial t}\right. & \left.+\frac{2 i \Omega_{1}^{2}}{\omega_{a b}}+\frac{4 i \Omega_{1} \Omega_{2}}{\omega_{a b}} \cos \left(2 \nu_{d} t\right)\right) \frac{\partial \Omega_{s}}{\partial t} \\
& +\Omega_{a}^{2}\left(1-\frac{2 \Omega_{1}^{2}}{\omega_{a b}^{2}}-\frac{2 \Omega_{1}^{2}}{\omega_{a b}^{2}} e^{2 i \nu_{d} t}\right) \Omega_{s}=0 .
\end{aligned}
$$

Equation (B10) has the structure

$$
\left(\frac{\partial}{\partial t}+\beta(t)\right) \frac{\partial \Omega_{s}}{\partial t}+\Omega_{a}^{2} f(t) \Omega_{s}=0 .
$$

Making a change of function

$$
\Omega_{s}=\tilde{\Omega}_{s} \exp \left(-\frac{1}{2} \int_{0}^{t} \beta\left(t^{\prime}\right) d t^{\prime}\right),
$$

we obtain

$$
\frac{\partial^{2} \tilde{\Omega}_{s}}{\partial t^{2}}+\left(\Omega_{a}^{2} f(t)-\frac{1}{2} \frac{\partial \beta(t)}{\partial t}-\frac{\beta^{2}(t)}{4}\right) \tilde{\Omega}_{s}=0 .
$$

Since $\beta(t)$ is a small perturbation, one can omit the term with $\beta^{2}(t)$. Using this formula, Eq. (B10) reduces to

$$
\begin{aligned}
& \frac{\partial^{2} \tilde{\Omega}_{s}}{\partial t^{2}}+\Omega_{a}^{2}\left[1-\delta-\delta \cos \left(2 \nu_{d} t\right)\right. \\
& \left.\quad+i \delta\left(\frac{2 \nu_{d} \omega_{a b}}{\Omega_{a}^{2}} \frac{\Omega_{2}}{\Omega_{1}}-1\right) \sin \left(2 \nu_{d} t\right)\right] \tilde{\Omega}_{s}=0,
\end{aligned}
$$

where

$$
\delta=\frac{2 \Omega_{1}^{2}}{\omega_{a b}^{2}} \ll 1
$$

is the dimensionless amplitude of modulation of collective oscillations. To suppress an unwanted Stark shift, one should choose

$$
\frac{\Omega_{2}}{\Omega_{1}}=\frac{\Omega_{a}^{2}}{2 \nu_{d} \omega_{a b}} .
$$

Then, Eq. (B14) becomes the equation of the parametric harmonic oscillator,

$$
\frac{\partial^{2} \tilde{\Omega}_{s}}{\partial t^{2}}+\Omega_{a}^{2}\left[1-\delta-\delta \cos \left(2 \nu_{d} t\right)\right] \tilde{\Omega}_{s}=0 .
$$

Near the parametric resonance $\nu_{d} \approx \Omega_{a}$, the seed pulse exponentially grows with time, $\Omega_{s}(t) \propto e^{G t}$, and gain per unit time is 


$$
G=\frac{\delta \cdot \Omega_{a}}{4}=\frac{\Omega_{a}}{2} \frac{\Omega_{1}^{2}}{\omega_{a b}^{2}}
$$

\section{Compensation of Stark shift by magnetic field}

Here, we assume that two-level atoms are described by the following model Hamiltonian:

$$
\begin{aligned}
\hat{H}= & {\left[\hbar \omega_{a}-\mu H_{z}(t)\right]|a\rangle\left\langle a\left|+\hbar \omega_{b}\right| b\right\rangle\langle b| } \\
& -\left(\mathbf{d}_{a b}|a\rangle\left\langle b\left|+\mathbf{d}_{b a}\right| b\right\rangle\langle a|\right) \mathbf{E}(t),
\end{aligned}
$$

implying that state $a$ has a magnetic moment $\mu$ along the $z$ axis that interacts with the $z$ component of the magnetic field. In addition, levels $a$ and $b$ are dipole coupled by the electric field and, e.g., $\mathbf{d}_{a b}=d(\hat{x}+i \hat{y})$. The magnetic field produces a time-dependent Zeeman shift of the level $a$. As a result, the atomic frequency in Eq. (A3) is replaced with $\omega_{a b} \rightarrow \omega_{a b}-\Omega_{H}(t)$, where

$$
\Omega_{H}(t)=\frac{\mu H_{z}(t)}{\hbar} .
$$

The magnetic field produces a similar effect as the Stark shift, and hence, they can compensate each other. Analysis of the previous section can be applied here, and it yields the same evolution equation (A18) for $\Omega_{s}$ but with

$$
\Delta \omega_{a b}(t)=\frac{2\left|\Omega^{\text {drive }}\right|^{2}}{\omega_{a b}}-\Omega_{H}
$$

Here, we assume that the atomic medium is driven by a strong linearly polarized laser pulse propagating along the $z$ axis,

$$
\Omega^{\mathrm{drive}}(t, z)=\Omega_{d} \cos \left(\nu_{d} t-k_{d} z\right),
$$

where $\nu_{d} \ll \omega_{a b}$ and $\Omega_{d} \ll \omega_{a b}$. Equations (A12) and (A13) yield that, under the influence of the driving field (which is turned on adiabatically), atomic population and coherence evolve as

$$
\begin{gathered}
\rho_{a a}^{\text {drive }}=\frac{\Omega_{d}^{2}}{\omega_{a b}^{2}-\nu_{d}^{2}} \cos ^{2}\left(\nu_{d} t-k_{d} z\right), \\
\rho_{a b}^{\text {drive }}=\frac{\Omega_{d}}{2}\left(\frac{e^{i\left(\nu_{d} t-k_{d} z\right)}}{\omega_{a b}+\nu_{d}}+\frac{e^{-i\left(\nu_{d} t-k_{d} z\right)}}{\omega_{a b}-\nu_{d}}\right) .
\end{gathered}
$$

In order to compensate for the Stark shift, the applied magnetic field should have the form

$$
\Omega_{H}(t, z)=\Omega_{m} \cos \left(2 \nu_{d} t-2 k_{d} z\right) .
$$

Taking into account Eqs. (A18) and (B20)-(B24), we obtain the following evolution equation for the superradiant pulse $\Omega_{s}$ :

$$
\begin{gathered}
{\left[\frac{\partial}{\partial t}+i \omega_{a b} \delta+i\left(\omega_{a b} \delta-\Omega_{m}\right) \cos \left(2 \nu_{d} t-2 k_{d} z\right)\right.} \\
\left.-\nu_{d} \delta \sin \left(2 \nu_{d} t-2 k_{d} z\right)\right]\left(c \frac{\partial}{\partial z}+\frac{\partial}{\partial t}\right) \Omega_{s} \\
+\Omega_{a}^{2}\left[1-4 \delta \cos ^{2}\left(\nu_{d} t-k_{d} z\right)\right] \Omega_{s}=0
\end{gathered}
$$

where

$$
\delta=\frac{\Omega_{d}^{2}}{\omega_{a b}^{2}} \ll 1
$$

is the dimensionless modulation amplitude of collective atomic oscillations.

\section{a. Gain per unit time: Reduction to Mathieu's equation}

To find the exact analytical solution of Eq. (B25), we first reduce it to Mathieu's equation. Equation (B25) has the structure

$$
\begin{aligned}
\left(\frac{\partial}{\partial t}\right. & \left.+i \omega_{a b} \delta+\beta\left(t-z / v_{d}\right)\right)\left(c \frac{\partial}{\partial z}+\frac{\partial}{\partial t}\right) \Omega_{s} \\
& +\Omega_{a}^{2} f\left(t-z / v_{d}\right) \Omega_{s}=0,
\end{aligned}
$$

where

$$
v_{d}=\frac{\nu_{d}}{k_{d}}
$$

is the phase velocity of the driving field along the $z$ axis. We look for the solution of Eq. (B27) in the form

$$
\Omega_{s}(t, z)=e^{i\left(\nu-\omega_{a b} \delta\right)(t-z / c)} \Omega_{s}(\xi),
$$

where $\xi=t-z / v_{d}$ and $\nu$ is complex. The imaginary part of $\nu$ gives gain per unit time $G$, provided that $\Omega_{s}(t, z)$ satisfies the proper initial condition. Namely, at $t=0$, the function $\Omega_{s}(0, z)$ should be finite at all $z$.

Plugging (B29) into Eq. (B27) yields

$$
\frac{\partial^{2} \Omega_{s}(\xi)}{\partial \xi^{2}}+[i \nu+\beta(\xi)] \frac{\partial \Omega_{s}(\xi)}{\partial \xi}+\frac{\Omega_{a}^{2} f(\xi)}{1-c / v_{d}} \Omega_{s}(\xi)=0 .
$$

Making a change of function

$$
\Omega_{s}(\xi)=\tilde{\Omega}_{s}(\xi) \exp \left(-\frac{1}{2} i \nu \xi-\frac{1}{2} \int_{0}^{\xi} \beta\left(\xi^{\prime}\right) d \xi^{\prime}\right),
$$

we obtain

$$
\frac{\partial^{2} \tilde{\Omega}_{s}}{\partial \xi^{2}}+\left(\frac{\Omega_{a}^{2} f(\xi)}{1-c / v_{d}}-\frac{1}{2} \frac{\partial \beta(\xi)}{\partial \xi}-\frac{1}{4}[i \nu+\beta(\xi)]^{2}\right) \tilde{\Omega}_{s}=0
$$

If the expression in (...) is a periodic function of $\xi$, then Eq. (B32) is known as Hill's equation.

Suppose that we find the solution of Eq. (B32) which grows exponentially, namely, 


$$
\tilde{\Omega}_{s}(\xi) \propto e^{G_{1} \xi} .
$$

Then, according to Eqs. (B29) and (B31), the function $\Omega_{s}(t, z)$ grows as

$$
\begin{aligned}
\Omega_{s}(t, z) \propto & \exp \left(\left[\frac{i \nu}{2}+G_{1}\right] t\right) \\
& \times \exp \left(\left[i \nu\left(\frac{1}{2}-\frac{v_{d}}{c}\right)-G_{1}\right] \frac{z}{v_{d}}\right) .
\end{aligned}
$$

$\Omega_{s}(t, z)$ satisfies the proper initial condition if $\Omega_{s}(0, z)$ remains finite at all $z$. This yields

$$
\operatorname{Im}(\nu)=\frac{2 c G_{1}}{2 v_{d}-c}
$$

Gain of the pulse per unit time is then given by

$$
G=G_{1}-\frac{\operatorname{Im}(\nu)}{2}=2 G_{1}\left(\frac{c-v_{d}}{c-2 v_{d}}\right) .
$$

Equation (B36) shows that there is no gain in the forward direction $\left(v_{d}=c\right)$.

Since $\beta(\xi)$ is a small perturbation, one can omit the term with $\beta^{2}(\xi)$ and write Eq. (B32) as

$$
\frac{\partial^{2} \tilde{\Omega}_{s}}{\partial \xi^{2}}+\left(\frac{\Omega_{a}^{2} f(\xi)}{1-c / v_{d}}+\frac{\nu^{2}}{4}-\frac{1}{2} \frac{\partial \beta(\xi)}{\partial \xi}-\frac{i \nu}{2} \beta(\xi)\right) \tilde{\Omega}_{s}=0 .
$$

Using this formula and taking into account that

$$
\begin{gathered}
\beta(\xi)=i\left(\omega_{a b} \delta-\Omega_{m}\right) \cos \left(2 \nu_{d} \xi\right)-\nu_{d} \delta \sin \left(2 \nu_{d} \xi\right), \\
f(\xi)=1-4 \delta \cos ^{2}\left(\nu_{d} \xi\right)
\end{gathered}
$$

one can reduce Eq. (B25) to

$$
\begin{aligned}
& \frac{\partial^{2} \tilde{\Omega}_{s}}{\partial \xi^{2}}+\left[\frac{\Omega_{a}^{2}}{1-c / v_{d}}(1-2 \delta)+\frac{\nu^{2}}{4}\right. \\
& \quad+\left(\nu_{d}^{2} \delta-\frac{2 \Omega_{a}^{2} \delta}{1-c / v_{d}}+\frac{1}{2} \nu\left(\omega_{a b} \delta-\Omega_{m}\right)\right) \cos \left(2 \nu_{d} \xi\right) \\
& \left.\quad+i \nu_{d}\left(\omega_{a b} \delta-\Omega_{m}+\frac{\nu \delta}{2}\right) \sin \left(2 \nu_{d} \xi\right)\right] \tilde{\Omega}_{s}=0,
\end{aligned}
$$

which can be written in the form of Mathieu's equation,

$$
\frac{\partial^{2} \tilde{\Omega}_{s}}{\partial \xi^{2}}+\tilde{\Omega}_{a}^{2}\left[1+\delta_{0} \cos \left(2 \nu_{d} \xi+\phi\right)\right] \tilde{\Omega}_{s}=0,
$$

where

$$
\begin{gathered}
\tilde{\Omega}_{a}^{2}=\frac{\Omega_{a}^{2}}{1-c / v_{d}}(1-2 \delta)+\frac{\omega^{2}}{4}, \\
\delta_{0}=\left[\left(\nu_{d}^{2} \delta-\frac{2 \Omega_{a}^{2} \delta}{1-c / v_{d}}+\frac{1}{2} \nu\left(\omega_{a b} \delta-\Omega_{m}\right)\right)^{2}\right. \\
\left.-\nu_{d}^{2}\left(\omega_{a b} \delta-\Omega_{m}+\frac{\nu \delta}{2}\right)^{2}\right]^{1 / 2} \frac{1}{\tilde{\Omega}_{a}^{2}}
\end{gathered}
$$

and $\phi$ is an irrelevant constant. Since the imaginary part of $\nu$ (gain) is small, one can treat $\nu$ in Eqs. (B42) and (B43) as real. With the same accuracy, one can disregard the term $2 \delta$ in Eq. (B42).

The condition of the first-order resonance $\nu_{d}=\tilde{\Omega}_{a}$ yields the following expression for the frequency of the most unstable mode:

$$
\nu= \pm 2 \sqrt{\nu_{d}^{2}-\frac{\Omega_{a}^{2}}{1-c / v_{d}}}
$$

For such a frequency, gain per unit time is

$$
\begin{aligned}
G= & 2 G_{1}\left(\frac{c-v_{d}}{c-2 v_{d}}\right)=2\left(\frac{c-v_{d}}{c-2 v_{d}}\right) \frac{\delta_{0} \nu_{d}}{4} \\
= & \frac{c-v_{d}}{c-2 v_{d}} \frac{\Omega_{a}}{2 \nu_{d} \sqrt{1-c / v_{d}}} \\
& \times\left[\delta^{2} \nu_{d}^{2}-\left(\omega_{a b} \delta-\Omega_{m} \pm 2 \delta \sqrt{\omega_{d}^{2}-\frac{\Omega_{a}^{2}}{1-c / v_{d}}}\right)^{2}\right]^{1 / 2} .
\end{aligned}
$$

Equation (B44) gives the following necessary condition of existence of the first-order parametric resonance,

$$
\nu_{d}^{2} \geq \frac{v_{d} \Omega_{a}^{2}}{v_{d}-c} .
$$

Equation (B45) predicts the following. If $c / v_{d}<1$ (which includes gain in the backward direction $v_{d}=-c$ or the case of uniform drive $k_{d}=0$, that is, $v_{d}=\infty$ ), then the optimum value of $\Omega_{m}$ which maximizes gain is given by

$$
\Omega_{m}=\omega_{a b} \delta \pm 2 \delta \sqrt{\nu_{d}^{2}-\frac{\Omega_{a}^{2}}{1-c / v_{d}}} .
$$

For such $\Omega_{m}$, gain is

$$
G=\frac{c-v_{d}}{c-2 v_{d}} \frac{\delta \cdot \Omega_{a}}{2 \sqrt{1-c / v_{d}}} .
$$

\section{b. Gain per unit length: Treatment in $t$, $z$ coordinates}

Here, we solve the original evolution equation (B25) for the field and find how unstable modes grow in space. Making in Eq. (B25) the change of function

$$
\Omega_{s} \rightarrow \Omega_{s} \exp \left[-i\left(t-\frac{z}{c}\right) \omega_{a b} \delta\right]
$$

we obtain

$$
\begin{aligned}
& \left(\frac{\partial}{\partial t}+\delta_{1} \nu_{d} \sin \left(2 \nu_{d} t-2 k_{d} z\right)+i \delta_{3} \nu_{d} \cos \left(2 \nu_{d} t-2 k_{d} z\right)\right) \\
& \quad \times\left(c \frac{\partial}{\partial z}+\frac{\partial}{\partial t}\right) \Omega_{s}+\Omega^{2}\left[1+\delta_{2} \cos \left(2 \nu_{d} t-2 k_{d} z\right)\right] \Omega_{s} \\
& =0
\end{aligned}
$$

where 


$$
\Omega=\Omega_{a} \sqrt{1-2 \delta}
$$

and

$$
\delta_{1}=-\delta, \quad \delta_{2}=-\frac{2 \delta}{1-2 \delta}, \quad \delta_{3}=\frac{1}{\nu_{d}}\left(\omega_{a b} \delta-\Omega_{m}\right)
$$

are small numbers.

We look for a solution for $\Omega_{s}$ in the form

$$
\Omega_{s}=\left(1+A e^{2 i\left(\nu_{d} t-k_{d} z\right)}\right) e^{i k z-i \nu t},
$$

where $A$ is a constant and $\nu$ is the detuning of $\Omega_{s}$ from the transition frequency $\omega_{a b}$. We assume that $\nu$ is real. Then, the imaginary part of $k$ gives gain $G_{\mathrm{L}}$ (absorption) per unit length of the mode with frequency $\nu$. During propagation of the seed pulse $\Omega_{s}$ through the medium, it grows as $\exp \left(G_{\mathrm{L}} z\right)$, where $G_{\mathrm{L}}=-\operatorname{Im}(k)$.

Substituting Eq. (B50) into Eq. (B49) and making the rotating wave approximation, that is, replacing

$$
\begin{gathered}
\cos \left(2 \nu_{d} t-2 k_{d} z\right) e^{ \pm 2 i\left(\nu_{d} t-k_{d} z\right)} \rightarrow \frac{1}{2}, \\
\sin \left(2 \nu_{d} t-2 k_{d} z\right) e^{ \pm 2 i\left(\nu_{d} t-k_{d} z\right)} \rightarrow \pm \frac{i}{2},
\end{gathered}
$$

and then eliminating $A$ yields the following equation for $k$ :

$$
\begin{aligned}
& {\left[\left(\nu-2 \nu_{d}\right)\left(c k-\nu-2 c k_{d}+2 \nu_{d}\right)+\Omega^{2}\right]\left[\nu(c k-\nu)+\Omega^{2}\right]} \\
& =\frac{1}{4}\left[\delta_{2} \Omega^{2}+\left(\delta_{1}-\delta_{3}\right) \nu_{d}(c k-\nu)\right] \\
& \quad \times\left[\delta_{2} \Omega^{2}-\left(\delta_{1}+\delta_{3}\right) \nu_{d}\left(c k-\nu-2 c k_{d}+2 \nu_{d}\right)\right] .
\end{aligned}
$$

Equation (B53) has the structure

$$
\left(k-k_{1}\right)\left(k-k_{2}\right)=H(k, \nu),
$$

where $k_{1}$ and $k_{2}$ are real numbers given by the roots of Eq. (B53) without the right-hand side, and

$$
\begin{aligned}
H(k, \nu)= & \frac{\left[\delta_{2} \Omega^{2}+\left(\delta_{1}-\delta_{3}\right) \nu_{d}(c k-\nu)\right]}{c^{2}\left[4\left(\nu-2 \nu_{d}\right) \nu+\left(\delta_{1}^{2}-\delta_{3}^{2}\right) \nu_{d}^{2}\right]} \\
& \times\left[\delta_{2} \Omega^{2}-\left(\delta_{1}+\delta_{3}\right) \nu_{d}\left(c k-\nu-2 c k_{d}+2 \nu_{d}\right)\right]
\end{aligned}
$$

is a small correction of the order of $\delta^{2}$. The formal solution of Eq. (B54) is

$$
k=\frac{1}{2}\left(k_{1}+k_{2}\right) \pm \sqrt{\frac{1}{4}\left(k_{1}-k_{2}\right)^{2}+H(k, \nu)} .
$$

Gain is maximum when $k_{1}=k_{2}$. This condition gives two equations for $k$ and $\nu$,

$$
\begin{gathered}
\left(\nu-2 \nu_{d}\right)\left(c k-\nu-2 c k_{d}+2 \nu_{d}\right)+\Omega^{2}=0, \\
\nu(c k-\nu)+\Omega^{2}=0,
\end{gathered}
$$

which yield

$$
\nu=\nu_{d} \pm \sqrt{\nu_{d}^{2}-\frac{\Omega^{2}}{1-c k_{d} / \nu_{d}}} .
$$

Plugging $k$ and $\nu$ into Eq. (B56), we obtain that gain per unit length is given by

$$
\begin{aligned}
G_{\mathrm{L}}= & \sqrt{-H(k, \nu)} \\
= & \frac{\Omega\left|\nu_{d}-c k_{d}\right|}{c \sqrt{4 \Omega^{2}+\left(\delta_{3}^{2}-\delta_{1}^{2}\right) \nu_{d}^{2}\left(1-c k_{d} / \nu_{d}\right)}} \\
& \times \sqrt{\left(\delta_{2}-\delta_{1}\right)^{2}-\left(\delta_{2} \sqrt{1+\frac{\Omega^{2}}{\nu_{d}\left(c k_{d}-\nu_{d}\right)}} \pm \delta_{3}\right)^{2}} .
\end{aligned}
$$

Plugging $\delta_{1}, \delta_{2}$, and $\delta_{3}$ into the above equation, we finally obtain for $\left(\delta_{3}^{2}-\delta_{1}^{2}\right) \nu_{d}^{2}\left(1-c k_{d} / \nu_{d}\right) \ll \Omega^{2}$

$$
\begin{gathered}
G_{\mathrm{L}} \approx \\
2 \frac{\left|\nu_{d}-c k_{d}\right|}{2 c}\left[\delta^{2}-\frac{1}{\nu_{d}^{2}}\left(\omega_{a b} \delta-\Omega_{m}\right.\right. \\
\left.\mp 2 \delta \sqrt{\left.\nu_{d}^{2}+\frac{\Omega^{2} \nu_{d}}{\left(c k_{d}-\nu_{d}\right)}\right)^{2}}\right]^{1 / 2} .
\end{gathered}
$$

The gain is maximum if the second term under the square root is equal to zero, which yields the following expression for the optimum magnetic field strength:

$$
\Omega_{m}=\frac{\Omega_{d}^{2}}{\omega_{a b}^{2}}\left(\omega_{a b} \mp 2 \sqrt{\nu_{d}^{2}+\frac{\Omega^{2} \nu_{d}}{\left(c k_{d}-\nu_{d}\right)}}\right) .
$$

The maximum gain is

$$
G_{\mathrm{L}} \approx \frac{\left|\nu_{d}-c k_{d}\right|}{2 c} \frac{\Omega_{d}^{2}}{\omega_{a b}^{2}} .
$$

Gain exists if Eq. (B59) yields real $\nu$. This result imposes a constraint on the driving field frequency,

$$
\nu_{d}^{2}>\frac{\nu_{d} \Omega_{a}^{2}}{\nu_{d}-c k_{d}},
$$

which coincides with Eq. (B46).

\section{Numerical simulations}

Next, we investigate numerically how the weak pulse evolves in time and space. As in the previous section, we assume that atoms are driven by linearly polarized light and that the Stark shift is compensated by applying an additional magnetic field. The total field, the total coherence, and population of the excited state are given by Eqs. (A4)-(A9) in which $\Omega^{\text {drive }}$ is a fixed function. In the presence of an additional magnetic field, shifting the level $a$, the evolution of $\rho_{a b}^{\text {drive }}$ and $\rho_{a a}^{\text {drive }}$ is governed by

$$
\dot{\rho}_{a b}^{\text {drive }}=-i\left(\omega_{a b}-\Omega_{H}\right) \rho_{a b}^{\text {drive }}+i \Omega^{\text {drive }}\left(1-2 \rho_{a a}^{\text {drive }}\right),
$$




$$
\dot{\rho}_{a a}^{\text {drive }}=i \Omega^{\text {drive }} \rho_{b a}^{\text {drive }}-i \Omega^{\text {drive* }} \rho_{a b}^{\text {drive }} .
$$

Equations for slowly varying envelopes of quantities associated with the superradiant field $\Omega_{s}(t, z), \rho_{a b}^{s}(t, z)$, and $\rho_{a a}^{s}(t, z)$ read

$$
\begin{gathered}
\dot{\rho}_{a b}^{s}=i \Omega_{H} \rho_{a b}^{s}+i \Omega_{s}\left(1-2 \rho_{a a}^{\mathrm{drive}}\right)-2 i \Omega^{\mathrm{drive}} \rho_{a a}^{s}, \\
i \dot{\rho}_{a a}^{s}+\omega_{a b} \rho_{a a}^{s}=\Omega_{s}\left(\rho_{a b}^{\mathrm{drive}}-\text { c.c. }\right)+\Omega^{\mathrm{drive}^{*}} \rho_{a b}^{s}, \\
\left(c \frac{\partial}{\partial z}+\frac{\partial}{\partial t}\right) \Omega_{s}=i \Omega_{a}^{2} \rho_{a b}^{s} .
\end{gathered}
$$

Here, we solve Eqs. (B65)-(B69) numerically. First, we assume that $\Omega^{\text {drive }}$ and $\Omega_{s}$ depend only on time, and $\Omega^{\text {drive }}$ is turned on adiabatically as

$\Omega^{\text {drive }}(t)=\frac{\Omega_{d}}{2}\left[1+\tanh \left(-3+0.2 \Omega_{a} t\right)\right] \sin \left(\nu_{d} t\right)$,

while $\Omega_{H}$ is chosen to compensate the Stark shift,

$$
\Omega_{H}(t)=\frac{2}{\omega_{a b}}\left[\Omega^{\text {drive }}(t)\right]^{2} .
$$

We solve equations with initial conditions $\rho_{a a}(0)=0$, $\rho_{b b}(0)=1, \rho_{a b}(0)=0$, and $\dot{\Omega}_{s}(0)=0$. In simulations, we take $\omega_{a b}=10.4 \Omega_{a}, \Omega_{d}=\Omega_{a}$, and $\nu_{d}$ is chosen to maximize gain. We find that gain is maximum for $\nu_{d}=0.990 \Omega_{a}$; that is, $\omega_{a b} / \nu_{d}=10.5$ is far from the multiphoton resonance. We plot the results of the numerical simulations in Fig. 7, which shows $\left|\Omega_{s}\right|$ as a function of time. The superradiant field undergoes exponentially growing oscillations, and gain per unit time is $G=0.0018 \Omega_{a}$.

Figure 8 shows gain per unit time $G$ for the optimal driving field frequency $\nu_{d}$ (corresponding to the parametric resonance) as a function of the strength of the driving field $\Omega_{d}$. Parameters are the same as for Fig. 7. The plot

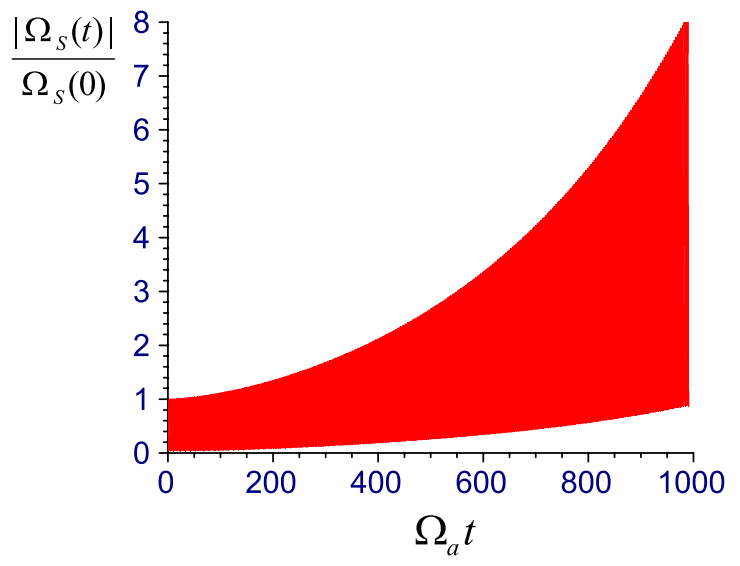

FIG. 7. Evolution of the superradiant pulse as a function of time obtained by numerical solution of Eqs. (B65)-(B71) with $\rho_{a a}(0)=0, \rho_{b b}(0)=1, \rho_{a b}(0)=0, \omega_{a b}=10.4 \Omega_{a}, \Omega_{d}=\Omega_{a}$, and $\nu_{d}=0.990 \Omega_{a}$.

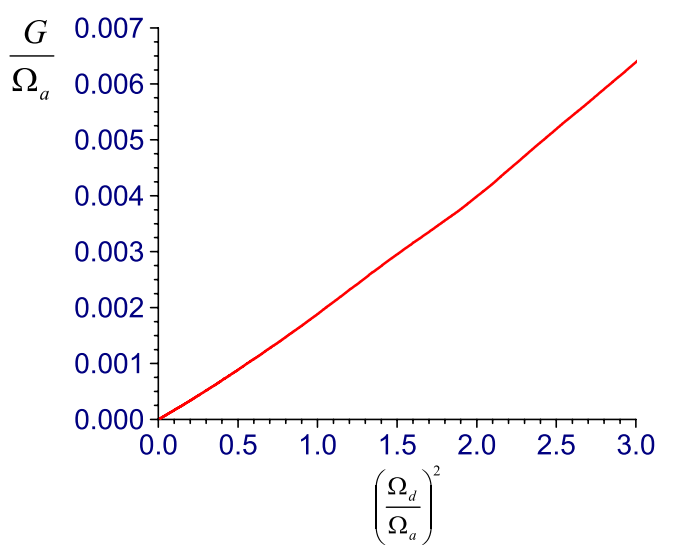

FIG. 8. Maximum gain per unit time as a function of $\Omega_{d}$ obtained by numerical solution of Eqs. (B65)-(B71) (solid line). Initially, all population is in the ground state $b$. In simulations, we take $\omega_{a b}=10.4 \Omega_{a}$, and $\nu_{d}$ is chosen to maximize the gain.

demonstrates that for small $\Omega_{d}$ gain is proportional to $\left|\Omega_{d}\right|^{2}$. The $\left|\Omega_{d}\right|^{2}$ dependence indicates that gain is not governed by the multiphoton resonance.

To demonstrate the importance of Stark-shift compensation, we calculate maximum gain as a function of $\Omega_{H}$. Full compensation of the Stark shift occurs at $\Omega_{H}^{\text {optimum }}$, as given by Eq. (B71). In Fig. 9, we plot maximum gain per unit time as a function of $\Omega_{H} / \Omega_{H}^{\text {optimum }}$. In numerical calculations, we take $\omega_{a b}=10.4 \Omega_{a}, \Omega_{d}=0.5 \Omega_{a}$, and $\nu_{d}$ is chosen to maximize gain in the vicinity of parametric resonance $\nu_{d} \approx \Omega_{a}$. The figure shows that gain is positive if $\Omega_{H}$ lies in a narrow interval near the optimum value. Outside this interval, the time-dependent Stark shift suppresses gain.

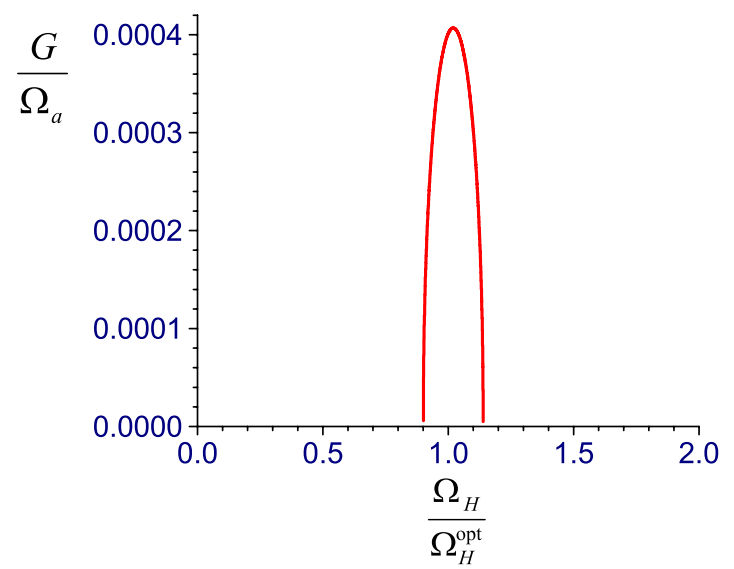

FIG. 9. Maximum gain per unit time as a function of the magnetic field amplitude, which shifts energy of the excited state $a$ and compensates for the time-dependent Stark shift of the $a-b$ transition. Results are obtained by numerical solution of Eqs. (B65)-(B69) with $\omega_{a b}=10.4 \Omega_{a}, \Omega_{d}=0.5 \Omega_{a}$, and an optimum value of $\nu_{d}$ that maximizes gain. 


\section{APPENDIX C: COUPLED PARAMETRIC OSCILLATORS}

Here, we consider two coupled harmonic oscillators described by the equations

$$
\begin{gathered}
\ddot{x}_{1}+\omega_{0}^{2} x_{1}-\Omega^{2} x_{2}=0, \\
\ddot{x}_{2}+\omega_{0}^{2} x_{2}-\Omega^{2} g(t) x_{2}-\Omega^{2} f(t)\left(x_{1}-x_{2}\right)=0,
\end{gathered}
$$

where $\omega_{0}$ is the frequency of the free oscillator, $\Omega \ll \omega_{0}$ is a coupling constant,

$$
f(t)=1+\delta \cos \left(\nu_{d} t\right)
$$

$\nu_{d} \ll \omega_{0}$ is the driving frequency, and $\delta \ll 1$ is a modulation amplitude. The function $f(t)$ describes the modulation of the coupling strength between oscillators. Such a modulation produces an unwanted time-dependent frequency shift of the second oscillator. To compensate such a shift, we additionally modulate the oscillator frequency by introducing the function $g(t)$.

Making a slowly varying amplitude approximation, that is, writing

$$
x_{1}=A_{1}(t) e^{i \omega_{0} t}, \quad x_{2}=A_{2}(t) e^{i \omega_{0} t},
$$

where $A_{1,2}(t)$ are slowly varying functions on the time scale $1 / \omega_{0}$, we obtain the following equations for $A_{1}(t)$ and $A_{2}(t)$ :

$$
\begin{gathered}
2 i \omega_{0} \dot{A}_{1}-\Omega^{2} A_{2}=0, \\
2 i \omega_{0} \dot{A}_{2}-\Omega^{2} g(t) A_{2}-\Omega^{2} f(t)\left(A_{1}-A_{2}\right)=0 .
\end{gathered}
$$

Taking the time derivative of both sides of Eq. (C5) and using Eq. (C6), we find

$$
\ddot{A}_{1}-i \frac{\Omega^{2}}{2 \omega_{0}}[f(t)-g(t)] \dot{A}_{1}+\frac{\Omega^{4}}{4 \omega_{0}^{2}} f(t) A_{1}=0 .
$$

Equation (C7) shows that the frequency shift is the dominant effect since it is proportional to $1 / \omega_{0}$, while the term producing parametric resonance is of the order of $1 / \omega_{0}^{2}$. If we compensate the unwanted frequency shift, that is, choose $g(t)=f(t)$, then Eq. (C7) reduces to the parametric harmonic oscillator (Mathieu) equation

$$
\ddot{A}_{1}+\frac{\Omega^{4}}{4 \omega_{0}^{2}}\left[1+\delta \cos \left(\nu_{d} t\right)\right] A_{1}=0,
$$

which yields exponentially growing oscillations in the vicinities of parametric resonances. In particular, the firstorder parametric resonance occurs at the driving frequency

$$
\nu_{d}=\frac{\Omega^{2}}{\omega_{0}} .
$$

This frequency is much smaller than the oscillator frequency $\omega_{0}$. Nevertheless, Eq. (C8) yields large gain,

$$
G=\frac{\delta \cdot \nu_{d}}{8},
$$

because $\nu_{d}$ corresponds to the first-order parametric resonance.

Equations (C1) and (C2) describe small oscillations of two pendulums connected by a spring in which modulation of the coupling strength is provided by an electrical feedback mechanism, as shown in Fig. 3(a). In this arrangement, pendulums and the spring are made out of conducting materials. A metallic sphere of capacitance $C$ is attached to the top of the second pendulum, while the upper end of the first pendulum slides without friction along the resistor $R$ of length $l_{R}$ connected to the ac voltage supply $V(t)=V_{0} \cos \left(\nu_{d} t\right)$. The middle point of the resistor is grounded (has zero electric potential). The electric potential of the pendulums $\Phi$, and thus the charge of the metallic sphere $Q=C \Phi$, is determined by the displacement $\phi_{1}$ of the first pendulum from equilibrium, $\Phi=V(t) l_{1} \phi_{1} / l_{R}$ [for notation, see Fig. 3(a)]. A charge $q$, placed at a fixed position, produces the Coulomb force $F=q Q / 4 \pi \varepsilon_{0} r^{2}$ on the second pendulum. One can write this force as $F=F_{0} \cos \left(\nu_{d} t\right) \phi_{1}$, where $F_{0}=$ $q C V_{0} l_{1} / 4 \pi \varepsilon_{0} l_{R} r^{2}$ is approximately constant. The force $F$ is proportional to $\phi_{1}$ and modulates the coupling strength between oscillators via the time dependence of $V(t)$ without producing an undesirable frequency shift.

The equation of the pendulum motion around the axis of rotation reads

$$
\frac{d(I \dot{\phi})}{d t}=\tau,
$$

where $I$ is the moment of inertia, $\dot{\phi}$ is the angular velocity, and $\tau$ is the torque on the pendulum. Applying this equation for pendulums 1 and 2, we obtain for a small deviation from equilibrium

$M L^{2} \ddot{\phi}_{1}=-M g L \phi_{1}+k l^{2}\left(\phi_{2}-\phi_{1}\right)$, $M L^{2} \ddot{\phi}_{2}=-M g L \phi_{2}-k l^{2}\left(\phi_{2}-\phi_{1}\right)+F_{0} l_{2} \cos \left(\nu_{d} t\right) \phi_{1}$,

where $k$ is the spring constant. Combining terms, one can rewrite these equations as

$$
\begin{gathered}
\ddot{\phi}_{1}+\omega_{0}^{2} \phi_{1}-\Omega^{2} \phi_{2}=0, \\
\ddot{\phi}_{2}+\omega_{0}^{2} \phi_{2}-\Omega^{2}\left[1+\delta \cos \left(\nu_{d} t\right)\right] \phi_{1}=0,
\end{gathered}
$$

where

$$
\begin{gathered}
\omega_{0}^{2}=\frac{g}{L}+\frac{k l^{2}}{M L^{2}}, \\
\Omega^{2}=\frac{k l^{2}}{M L^{2}},
\end{gathered}
$$

and

$$
\delta=\frac{F_{0} l_{2}}{k l^{2}}
$$




\section{APPENDIX D: GROWTH OF ATOMIC POPULATION IN THE QASER}

Here, we derive Eq. (21), describing the growth of the excited-state population $\rho_{a a}$. Omitting spatial dependence, Eq. (5) yields

$$
\rho_{a b}(t)=-i \frac{\dot{\Omega}_{s}(t)}{\Omega_{a}^{2}}
$$

Plugging in $\Omega_{s}(t)=\Omega_{s}(0) \exp (G t)$, we find that the atomic coherence grows as

$$
\rho_{a b}(t)=\rho_{a b}(0) e^{G t},
$$

where $\rho_{a b}(0)$ is the initial coherence generated by the seed superradiant pulse. In the weak excitation limit $\rho_{a a}(t) \approx$ $\left|\rho_{a b}(t)\right|^{2}$ and using Eqs. (D2) and (31), we finally obtain

$$
\rho_{a a}(t) \approx\left|\rho_{a b}(0)\right|^{2} \exp \left(\frac{\sqrt{2}}{3} \frac{\Omega_{d}^{2}}{\omega_{a b}^{2}} \Omega_{a} t\right) .
$$

Atomic excitation can start to grow out of the vacuum fluctuations, or the growth can be triggered by the seed pulse $\Omega_{s}(0)$. This growth can be understood by considering the excitation of a pendulum with periodically modulated frequency. The equation of motion for such a parametric pendulum reads

$$
\ddot{\phi}+\omega_{0}^{2}\left[1+\delta \sin \left(\nu_{d} t\right)\right] \phi=0,
$$

where $\phi$ is a small angle describing the deviation from equilibrium and $\delta \ll 1$ is the modulation amplitude. The frequency modulation can be produced by periodically changing the length of the pendulum.

If initially the pendulum is not excited, that is, $\phi(0)=\dot{\phi}(0)=0$, then Eq. (D4) yields the null solution

$$
\phi(t)=0 .
$$

Hence, in order to amplify pendulum oscillations, we should first deposit a small amount of energy, e.g., shift the pendulum from its equilibrium position by some angle $\phi_{0}$. This is analogous to $\rho_{a b}(0)$ in Eq. (D2). In the zero-order approximation in parameter $\delta$, we then find

$$
\phi(t)=\phi_{0} \cos \left(\omega_{0} t\right) .
$$

Plugging (D6) back into Eq. (D4), we obtain in first order the equation of the forced harmonic oscillator,

$$
\ddot{\phi}+\omega_{0}^{2} \phi=\frac{1}{2} \omega_{0}^{2} \phi_{0} \delta\left(\sin \left[\left(\nu_{d}-\omega_{0}\right) t\right]-\sin \left[\left(\omega_{0}+\nu_{d}\right) t\right]\right) .
$$

For $\nu_{d}=2 \omega_{0}$, the first term in the right-hand side of Eq. (D7) acts as a driving force having resonant frequency $\omega_{0}$, which yields growth of the pendulum oscillations. However, the amplitude of the driving force is proportional to $\phi_{0}$, and thus, it would vanish unless the pendulum is initially excited.

\section{APPENDIX E: ELECTRONIC CIRCUIT ANALOG OF THE QASER}

Here, we discuss a realistic electronic circuit model that demonstrates the QASER amplification mechanism and that can be set up in an undergraduate electronics laboratory. The model consists of two LC circuits, as shown in Fig. 10, which are weakly coupled by a capacitor $C_{0}$ and a multiplier M. The output voltage $V_{\text {out }}$ of the multiplier is proportional to the product of the two input voltages $V(t)$ and $V_{1}: V_{\text {out }}=\kappa V(t) V_{1}$, where $\kappa$ is the gain of the multiplier and $V(t)=V_{0} \sin \left(\nu_{d} t\right)$ is the voltage produced by a function generator. We assume that the multiplier has negligible capacitance and high input resistance, so one can disregard its input current. In a realistic model, inductors have internal capacitance and resistance. As a consequence, in the electronic scheme we add resistors $R$ connected in series with the inductors and a capacitance connected in parallel.

Let the electric charges of the circuit capacitors be $Q_{0}$, $Q_{1}$, and $Q_{2}$. Applying Kirchhoff's laws to the system, we obtain (for notation, see Fig. 10)

$$
L_{1}\left(\ddot{Q}_{1}+\dot{I}_{0}\right)+R\left(\dot{Q}_{1}+I_{0}\right)+\frac{Q_{1}}{C_{1}}=0,
$$

$$
\begin{aligned}
& -\frac{Q_{2}}{C_{2}}+L_{2} \dot{I}_{3}-\kappa \frac{Q_{1}}{C_{1}} V(t)+R I_{3}=0, \\
& L_{2} \ddot{I}_{3}+R \dot{I}_{3}+\frac{1}{C_{3}}\left(I_{3}+\dot{Q}_{2}-I_{0}\right)=0,
\end{aligned}
$$

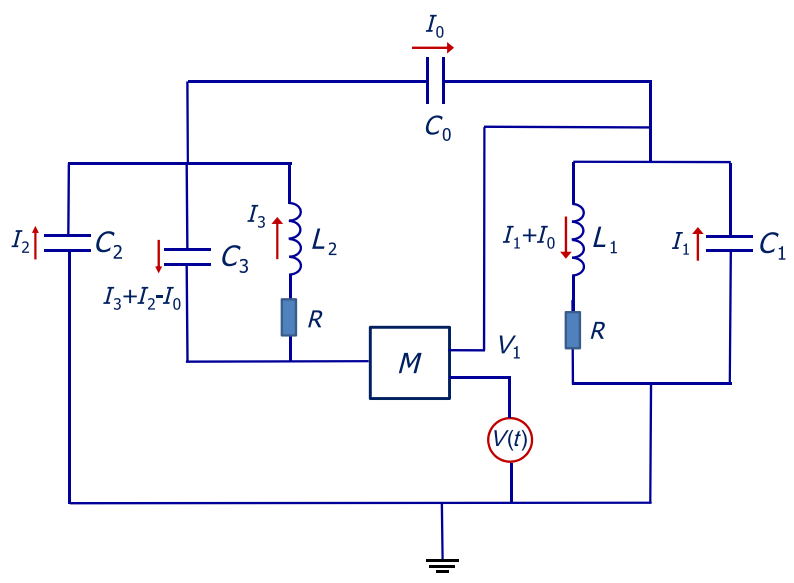

FIG. 10. Electronic circuit analog of the QASER. Two LC circuits weakly coupled by a capacitor $C_{0}$ correspond to the atoms and to the field. Modulation of the coupling strength is provided by a feedback mechanism in which voltage $V_{1}$ from the capacitor $C_{1}$ is applied to the input terminal of the multiplier $M$; its output voltage $V_{\text {out }}$ is proportional to the product of the two input voltages $V(t)$ and $V_{1}$, where $V(t)=V_{0} \sin \left(\nu_{d} t\right)$ is the voltage produced by a function generator. 


$$
\frac{Q_{2}}{C_{2}}+\frac{Q_{0}}{C_{0}}=\frac{Q_{1}}{C_{1}} .
$$

To demonstrate gain produced by driving the system at low frequency, we solve Eqs. (E1)-(E4) numerically for the following realistic parameters:

$$
\begin{gathered}
C_{0}=15 \mathrm{pF}, \quad C_{1}=200 \mathrm{pF}, \quad C_{2}=C_{3}=100 \mathrm{pF}, \\
L_{1}=L_{2}=4 \mathrm{mH}, \quad R=10 \Omega .
\end{gathered}
$$

We assume that initially there is no current through the inductors; the capacitor $C_{1}$ has charge $Q_{1}(0)$, while $Q_{2}(0)=0.035 Q_{1}(0)$. If there is no drive, that is, $V_{0}=0 \mathrm{~V}$, the system undergoes exponentially decaying oscillations, as shown in Fig. 11. Because of the coupling between two LC circuits, the system's high-frequency mode is split into $\omega_{1}=165.1 \mathrm{kHz}$ and $\omega_{2}=177.9 \mathrm{kHz}$. As a result, there is beating of the signal at the frequency difference $\Delta \omega=\omega_{2}-\omega_{1}=12.8 \mathrm{kH} z$.

Figure 12 shows the evolution of the charge $Q_{2}(t)$ of the capacitor $C_{2}$ if the circuit is driven with frequency $\nu_{d}=$ $\Delta \omega=12.8 \mathrm{kHz}$ and amplitude $\kappa V_{0}=0.1$. The system undergoes exponentially growing oscillations, and hence, the high-frequency modes $\omega_{1}$ and $\omega_{2}$ are amplified by the low-frequency drive. In Fig. 13, we plot gain per unit time as a function of the driving frequency $\nu_{d}$ for the drive amplitude $\kappa V_{0}=0.1$. When $\nu_{d}$ is equal to double the normal mode frequencies, that is, $\nu_{d}=330.2 \mathrm{kHz}$ and $355.8 \mathrm{kHz}$, the system experiences the first-order parametric resonances and gain has peaks. In addition, when $\nu_{d}$ is equal to the frequency difference between the two normal modes, that is, $\nu_{d}=\Delta \omega=12.8 \mathrm{kH} z$, there is also strong first-order parametric resonance that exemplifies the QASER amplification mechanism. It is interesting to note that, in the present model, the value of gain at the low driving frequency $\nu_{d}=\Delta \omega$ is greater than those at the high frequencies.

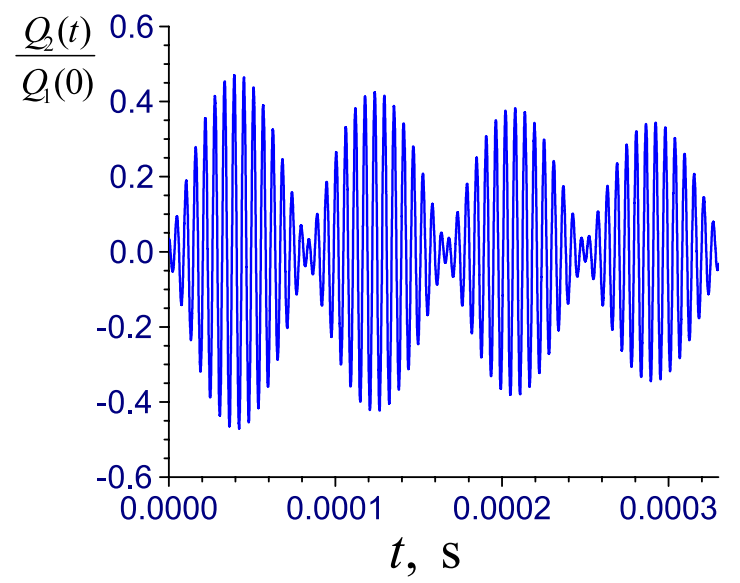

FIG. 11. Evolution of the charge $Q_{2}(t)$ of the capacitor $C_{2}$ for the electronic circuit of Fig. 10 with no drive. The circuit parameters are given by Eqs. (E5) and (E6).

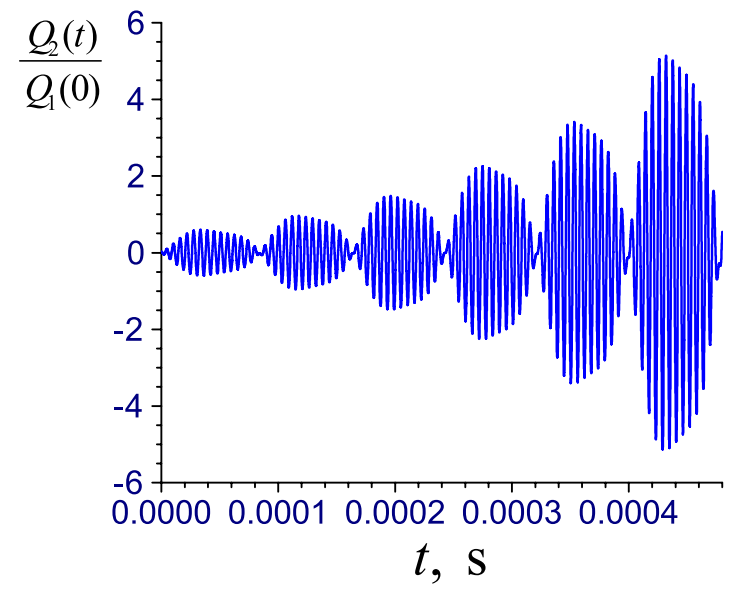

FIG. 12. The same as in Fig. 11, but now the circuit is driven by frequency $\nu_{d}=12.8 \mathrm{kHz}$ and amplitude $\kappa V_{0}=0.1$.

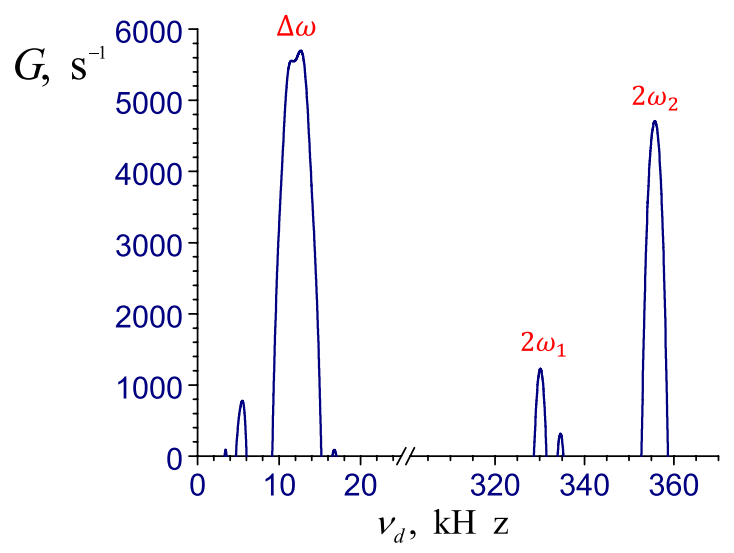

FIG. 13. Gain per unit time as a function of the driving frequency $\nu_{d}$ for the drive amplitude $\kappa V_{0}=0.1$. The circuit parameters are given by Eqs. (E5) and (E6).

\section{APPENDIX F: POSSIBLE EXPERIMENTAL REALIZATION OF THE QASER}

Here, we discuss possible experiments that can demonstrate light amplification by collective parametric resonance. To make the QASER work, we must compensate the time dependence of the ac Stark shift produced by the driving field for a particular atomic transition. Our analysis shows that such compensation, in the general case, does not suppress the transition modulation, which can lead to gain at high frequencies.

In many-level systems, one can compensate the unwanted Stark shift $\Delta \omega_{a b}(t)$, e.g., by driving atoms with a specific frequency or with a properly chosen elliptical polarization. We discuss these possibilities in the subsequent sections.

\section{Driving with specific frequency}

Because the contribution to $\Delta \omega_{a b}(t)$ from different levels can have opposite signs, one can compensate the Stark 
shift by a proper choice of the driving field frequency. To be specific, we consider gas of neutral He atoms that have energy levels as shown in Fig. 14. Initially, atoms are in ground state $1{ }^{1} \mathrm{~S}_{0}$, and driven by a linearly polarized field $E^{\text {drive }}(t)=E_{d} \cos \left(\nu_{d} t-k_{d} z\right.$ ), which couples dipoleallowed transitions, as shown in Fig. 14. The timedependent Stark shift of the $2{ }^{1} \mathrm{P}_{1}-1{ }^{1} \mathrm{~S}_{0}(a-b)$ transition is given by the following expression:

$$
\Delta \omega_{a b}(t)=\frac{\left|E^{\text {drive }}(t)\right|^{2}}{\hbar^{2}}\left(\frac{2 \omega_{a b}\left|d_{a b}\right|^{2}}{\omega_{a b}^{2}-\nu_{d}^{2}}-\sum_{m \neq a, b} \frac{\omega_{m a}\left|d_{a m}\right|^{2}}{\omega_{a m}^{2}-\nu_{d}^{2}}\right),
$$

where $\omega_{m a}=\omega_{m}-\omega_{a} . \Delta \omega_{a b}(t)$ can be made zero if the driving-field frequency has a proper value. Since the terms under the sum in Eq. (F1) are typically larger than the first term, one should choose $\nu_{d}$ to lie between two excited levels (see Fig. 14). In this case, the terms under the sum can compensate each other along with the first term.

For the 58.4-nm $2{ }^{1} \mathrm{P}_{1}-1{ }^{1} \mathrm{~S}_{0}$ transition $(\gamma=$ $\left.1.8 \times 10^{9} \mathrm{~s}^{-1}\right)$ and atomic density $n=1.6 \times 10^{20} \mathrm{~cm}^{-3}$, which corresponds to the helium pressure of $6.6 \mathrm{~atm}$ at room temperature $T=300 \mathrm{~K}$, the collective atomic frequency is

$$
\Omega_{a}=\sqrt{\frac{3 c n \lambda_{a b}^{2} \gamma}{8 \pi}}=1.87 \times 10^{14} \mathrm{~s}^{-1} .
$$

The atom-atom collision frequency is given by

$$
\nu_{\text {coll }}=V_{\mathrm{th}} n \sigma,
$$

where the collisional cross section is

$$
\sigma \approx a_{B}^{2} m^{4}=0.45 \times 10^{-15} \mathrm{~cm}^{2} .
$$

$m$ is the principal quantum number of the excited atomic state, and $a_{B}$ is the Bohr radius. For room temperature, atomic thermal velocity is

$$
V_{\mathrm{th}}=\sqrt{\frac{k_{B} T}{M}}=7.87 \times 10^{4} \mathrm{~cm} / \mathrm{s},
$$

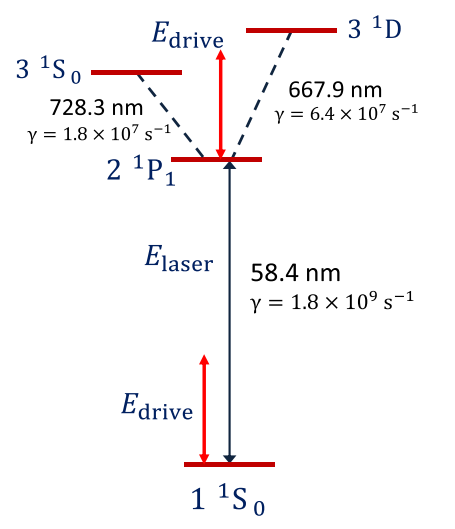

FIG. 14. Energy-level diagram of a He atom. which yields

$$
\nu_{\text {coll }}=V_{\mathrm{th}} n \sigma=5.7 \times 10^{9} \mathrm{~s}^{-1} .
$$

To fulfill the condition of collective parametric resonance, the driving-field frequency should be

$$
\nu_{d}>\frac{\Omega_{a}}{\sqrt{2}}
$$

which is readily satisfied in the present scheme. For driving-field intensity $I=10^{14} \mathrm{~W} / \mathrm{cm}^{2}$ (which is below the ionization threshold for He atoms), the Rabi frequency is

$$
\Omega_{d}=c \sqrt{\frac{3 \pi I \gamma_{a b}}{\hbar \omega_{a b}^{3}}}=6.6 \times 10^{14} \mathrm{~s}^{-1},
$$

and gain per unit time in the backward direction can be estimated [according to Eq. (31)] as

$$
G \approx \frac{\Omega_{a}}{3 \sqrt{2}} \frac{\Omega_{d}^{2}}{\omega_{a b}^{2}}=2 \times 10^{10} \mathrm{~s}^{-1},
$$

which is larger than collisional decoherence (this implies applicability of the present analysis). For the wavelength of the driving field, $\lambda \approx 700 \mathrm{~nm}$, Eq. (32) yields that gain per unit length is

$$
G_{L} \approx \frac{\nu_{d}}{c} \frac{\Omega_{d}^{2}}{\omega_{a b}^{2}}=30 \mathrm{~cm}^{-1},
$$

which is large enough to achieve one path light conversion into high-frequency radiation.

\section{Driving with elliptically polarized light}

One can also suppress the time dependence of the ac Stark shift by a proper choice of the elliptical polarization of the driving field. This can be understood from a threelevel model, as shown in Fig. 15. In the model, the ground state $b$ is dipole coupled with states $a$ and $c$. For example, the ground state can be an $s$ state, while levels $a$ and $c$ are $p_{x}$ and $p_{y}$ orbitals. We assume that the driving field $\mathbf{E}^{\text {drive }}$ couples both transitions, namely, the $x$ component of $\mathbf{E}^{\text {drive }}$ drives the $a-b$ transition, while the $y$ component drives the $c-b$ transition. Our goal is to compensate the ac Stark

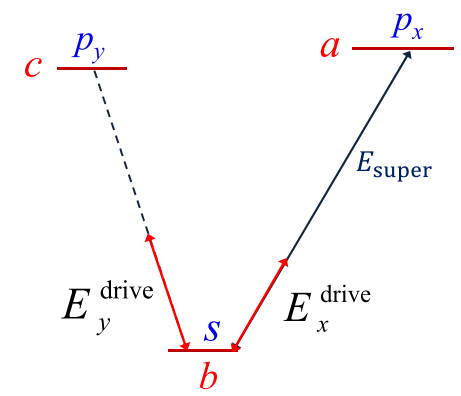

FIG. 15. Three-level model of the QASER. 
shift of the $a-b$ transition by a proper choice of elliptical polarization of $\mathbf{E}^{\text {drive }}$. The time-dependent Stark shift of the $a-b$ transition is given by the following expression:

$$
\begin{aligned}
\hbar^{2} \Delta \omega_{a b}(t)= & \frac{2 \omega_{a b}\left|d_{a b}\right|^{2}}{\omega_{a b}^{2}-\nu_{d}^{2}}\left|E_{x}^{\text {drive }}(t)\right|^{2} \\
& +\frac{\omega_{c b}\left|d_{c b}\right|^{2}}{\omega_{c b}^{2}-\nu_{d}^{2}}\left|E_{y}^{\text {drive }}(t)\right|^{2},
\end{aligned}
$$

which can be made time independent if we choose $E_{x}^{\text {drive }}=$ $A \cos \left(\nu_{d} t-k_{d} z\right) \quad$ and $\quad E_{y}^{\text {drive }}=B \sin \left(\nu_{d} t-k_{d} z\right) \quad$ with proper values of the amplitudes $A$ and $B$. Despite the compensation of the Stark-shift time dependence, the strength of the $a-b$ transition is yet modulated by the driving field, leading to gain at high frequencies. We show this effect in Appendix $G$ for the three-level model.

Real atoms have many levels, and many of them contribute to the Stark shift. Nevertheless, the mechanism of the ac Stark-shift compensation remains the same. In the presence of an external electric field $\mathbf{E}$, the Stark shift of the various magnetic sublevels of a particular electronic state may be expressed in terms of scalar and tensor polarizabilities, $\alpha_{0}$ and $\alpha_{2}$, which can be measured experimentally. If the atomic hyperfine structure is neglected, the frequency shift of a particular magnetic sublevel may be expressed in terms of its total angular momentum $J$ and the projection $m_{J}$ along the quantization axis $x$ as [44]

$\Delta \omega\left(J, m_{J}\right)=-\frac{1}{2} \alpha_{0} E^{2}-\frac{1}{4} \alpha_{2} \frac{3 m_{J}^{2}-J(J+1)}{J(2 J-1)}\left(3 E_{x}^{2}-E^{2}\right)$.

If the propagating driving field has $E_{x}$ and $E_{y}$ components, then Eq. (F12) yields

$$
\begin{aligned}
\Delta \omega\left(J, m_{J}\right)= & -\frac{1}{2}\left(\alpha_{0}+\alpha_{2} \frac{3 m_{J}^{2}-J(J+1)}{J(2 J-1)}\right) E_{x}^{2} \\
& -\frac{1}{2}\left(\alpha_{0}-\frac{1}{2} \alpha_{2} \frac{3 m_{J}^{2}-J(J+1)}{J(2 J-1)}\right) E_{y}^{2} .
\end{aligned}
$$

Equation (F13) shows that by a proper choice of time dependence of $E_{x}$ and $E_{y}$, one can make the Stark shift time independent.

Let us consider the $5 \mathrm{~s}^{2} \mathrm{~S}_{1 / 2}-5 \mathrm{p}{ }^{2} \mathrm{P}_{3 / 2}$ transition (D2 line) of the $\mathrm{Rb}$ atom $(\lambda=780.0 \mathrm{~nm})$ as the light amplification transition. Then, Eq. (F13) gives

$$
\begin{aligned}
\Delta \omega\left(5 s^{2} S_{1 / 2}\right)= & -\frac{1}{2} \alpha_{0}\left(5 s^{2} S_{1 / 2}\right)\left(E_{x}^{2}+E_{y}^{2}\right), \\
\Delta \omega\left(5 p^{2} P_{3 / 2}\right)= & -\frac{1}{2} \alpha_{0}\left(5 p^{2} P_{3 / 2}\right)\left(E_{x}^{2}+E_{y}^{2}\right) \\
& -\frac{1}{2} \alpha_{2}\left(5 p^{2} P_{3 / 2}\right)\left[m_{J}^{2}-\frac{5}{4}\right]\left(E_{x}^{2}-\frac{E_{y}^{2}}{2}\right),
\end{aligned}
$$

and the Stark shift of the transition frequency is

$$
\begin{aligned}
\Delta \omega= & \Delta \omega\left(5 p^{2} P_{3 / 2}\right)-\Delta \omega\left(5 s^{2} S_{1 / 2}\right) \\
= & -\frac{1}{2}\left(\alpha_{0}\left(5 p^{2} P_{3 / 2}\right)-\alpha_{0}\left(5 s^{2} S_{1 / 2}\right)\right)\left(E_{x}^{2}+E_{y}^{2}\right) \\
& -\frac{1}{2} \alpha_{2}\left(5 p^{2} P_{3 / 2}\right)\left[m_{J}^{2}-\frac{5}{4}\right]\left(E_{x}^{2}-\frac{E_{y}^{2}}{2}\right) .
\end{aligned}
$$

To make $\Delta \omega$ time independent, one should choose $E_{x}$ and $E_{y}$ such that

$$
\alpha_{x} E_{x}^{2}+\alpha_{y} E_{y}^{2}=\text { const, }
$$

where

$$
\begin{gathered}
\alpha_{x}=\alpha_{0}\left(5 p^{2} P_{3 / 2}\right)-\alpha_{0}\left(5 s^{2} S_{1 / 2}\right)+\alpha_{2}\left(5 p^{2} P_{3 / 2}\right)\left[m_{J}^{2}-\frac{5}{4}\right], \\
(\mathrm{F} 18) \\
\alpha_{y}=\alpha_{0}\left(5 p^{2} P_{3 / 2}\right)-\alpha_{0}\left(5 s^{2} S_{1 / 2}\right) \\
-\frac{1}{2} \alpha_{2}\left(5 p^{2} P_{3 / 2}\right)\left[m_{J}^{2}-\frac{5}{4}\right] .
\end{gathered}
$$

This result, e.g., is achieved for elliptically polarized light,

$$
\begin{aligned}
& E_{x}=E_{0 x} \cos \left(\nu_{d} t-k_{d} z\right), \\
& E_{y}=E_{0 y} \sin \left(\nu_{d} t-k_{d} z\right),
\end{aligned}
$$

with

$$
\frac{E_{0 x}^{2}}{E_{0 y}^{2}}=\frac{\alpha_{y}}{\alpha_{x}}
$$

For the $5 \mathrm{~s}{ }^{2} \mathrm{~S}_{1 / 2}-5 \mathrm{p}{ }^{2} \mathrm{P}_{3 / 2}$ transition of $\mathrm{Rb}$, the experimental values are $\alpha_{0}\left(5 p^{2} P_{3 / 2}\right)-\alpha_{0}\left(5 s^{2} S_{1 / 2}\right)=136 \mathrm{kHz} /$ $(\mathrm{kV} / \mathrm{cm})^{2}$ and $\alpha_{2}\left(5 p^{2} P_{3 / 2}\right)=-40 \mathrm{kHz} /(\mathrm{kV} / \mathrm{cm})^{2}$ [45]. Then, Eq. (F22) gives

$$
\frac{E_{0 x}^{2}}{E_{0 y}^{2}}=\frac{136+20\left[m_{J}^{2}-\frac{5}{4}\right]}{136-40\left[m_{J}^{2}-\frac{5}{4}\right]} .
$$

For example, for $m_{J}=1 / 2$ we obtain that the Stark shift is independent of time if

$$
\frac{E_{0 x}^{2}}{E_{0 y}^{2}}=0.66,
$$

while for $m_{J}=3 / 2$,

$$
\frac{E_{0 y}^{2}}{E_{0 x}^{2}}=0.62 .
$$

For the 780.0-nm $5 \mathrm{~s} \quad{ }^{2} \mathrm{~S}_{1 / 2}-5 \mathrm{p} \quad{ }^{2} \mathrm{P}_{3 / 2}$ transition $\left(\gamma=3.81 \times 10^{7} \mathrm{~s}^{-1}, \omega_{a b}=2.4 \times 10^{15} \mathrm{~s}^{-1}\right)$ and atomic density $n=10^{16} \mathrm{~cm}^{-3}$, the collective atomic frequency is

$$
\Omega_{a}=\sqrt{\frac{3 c n \lambda_{a b}^{2} \gamma}{8 \pi}}=2.9 \times 10^{12} \mathrm{~s}^{-1} .
$$

The atom-atom collision frequency is given by

$$
\nu_{\text {coll }}=V_{\text {th }} n \sigma,
$$


where the collisional cross section is

$$
\sigma \approx a_{B}^{2} m^{4}=1.7 \times 10^{-14} \mathrm{~cm}^{2}
$$

and $m=5$ is the principal quantum number of the excited atomic state. For temperature $T=500 \mathrm{~K}$, we obtain for the atomic thermal velocity,

$$
V_{\mathrm{th}}=\sqrt{\frac{k_{B} T}{M}}=2.2 \times 10^{4} \mathrm{~cm} / \mathrm{s},
$$

which yields

$$
\nu_{\text {coll }}=V_{\mathrm{th}} n \sigma=3.7 \times 10^{6} \mathrm{~s}^{-1} .
$$

The Doppler broadening is given by

$$
\Delta \nu_{\mathrm{D}}=\frac{V_{\mathrm{th}}}{c} \omega_{a b}=1.8 \times 10^{9} \mathrm{~s}^{-1} .
$$

If we choose the driving-field intensity $I=$ $10^{12} \mathrm{~W} / \mathrm{cm}^{2}$, then the driving-field Rabi frequency is

$$
\Omega_{d}=c \sqrt{\frac{3 \pi I \gamma_{a b}}{\hbar \omega_{a b}^{3}}}=4.7 \times 10^{14} \mathrm{~s}^{-1},
$$

and gain per unit time in the backward direction can be estimated as

$$
G \approx \frac{\Omega_{a}}{3 \sqrt{2}} \frac{\Omega_{d}^{2}}{\omega_{a b}^{2}}=2.5 \times 10^{10} \mathrm{~s}^{-1},
$$

which is much larger than collisional decoherence and Doppler broadening. One can drive $\mathrm{Rb}$ atoms with a commercial $\mathrm{CO}_{2}$ laser having wavelength $\lambda_{d} \approx 10.6 \mu \mathrm{m}$ $\left(\nu_{d}=1.8 \times 10^{14} \mathrm{~s}^{-1}\right)$. For such a driving field, the gain per unit length is estimated as

$$
G_{L} \approx \frac{\nu_{d}}{c} \frac{\Omega_{d}^{2}}{\omega_{a b}^{2}}=221 \mathrm{~cm}^{-1} .
$$

A similar experiment can be done in $\mathrm{Na}$ vapor. By driving $\mathrm{Na}$ atoms with a $\mathrm{CO}_{2}$ infrared laser at wavelength $10.6 \mu \mathrm{m}$ and choosing the proper elliptical polarization of the driving beam, one can achieve generation of yellow light produced by the sodium $\mathrm{D}$ line with wavelength $589 \mathrm{~nm}$ in a transition from the $3 p$ to the $3 s \mathrm{Na}$ level.

\section{APPENDIX G: THREE-LEVEL MODEL OF THE QASER}

Here, we consider a more realistic three-level model of the QASER shown in Fig. 15. In the model, the ground state $b$ is dipole coupled with states $a$ and $c$. For example, the ground state can be an $s$ state, while $a$ and $c$ states are $p_{x}$ and $p_{y}$ orbitals. The total electric field

$$
\mathbf{E}=\mathbf{E}^{\text {drive }}+\mathbf{E}^{\text {super }}
$$

is a sum of a low-frequency drive $\mathbf{E}^{\text {drive }}$, which we assume couples both transitions, and a weak high-frequency superradiant field $\mathbf{E}^{\text {super }}$ that couples only the $a-b$ transition. For example, this can be the case if $\mathbf{E}^{\text {drive }}$ has both $x$ and $y$ components, while $\mathbf{E}^{\text {super }}$ is linearly polarized along the $x$ axis.

For the present model, the Schrödinger equation yields the following evolution equations for the probability amplitudes $C_{m}$ to find atoms at the level $m=a, b, c$ (we put $\omega_{b}=0$ ):

$$
\begin{gathered}
\dot{C}_{a}+i \omega_{a b} C_{a}=\frac{i}{\hbar}\left[\mathbf{E}^{\text {drive }}+\mathbf{E}^{\text {super }}\right] \mathbf{d}_{a b} C_{b}, \\
\dot{C}_{c}+i \omega_{c b} C_{c}=\frac{i}{\hbar} \mathbf{E}^{\text {drive }} \mathbf{d}_{c b} C_{b},
\end{gathered}
$$

$$
\dot{C}_{b}=\frac{i}{\hbar}\left[\mathbf{E}^{\text {drive }}+\mathbf{E}^{\text {super }}\right] \mathbf{d}_{b a} C_{a}+\frac{i}{\hbar} \mathbf{E}^{\text {drive }} \mathbf{d}_{b c} C_{c},
$$

where $\omega_{a b}=\omega_{a}-\omega_{b}, \omega_{c b}=\omega_{c}-\omega_{b}$, and $\mathbf{d}_{n m}$ are the transition dipole matrix elements.

Introducing Rabi frequencies

$$
\begin{aligned}
& \boldsymbol{\Omega}_{c b}^{\text {drive }}=\frac{1}{\hbar} \mathbf{E}^{\text {drive }} \cdot \mathbf{d}_{c b}, \\
& \boldsymbol{\Omega}_{a b}^{\text {drive }}=\frac{1}{\hbar} \mathbf{E}^{\text {drive }} \cdot \mathbf{d}_{a b}, \\
& \boldsymbol{\Omega}^{\text {super }}=\frac{1}{\hbar} \mathbf{E}^{\text {super }} \cdot \mathbf{d}_{a b},
\end{aligned}
$$

we obtain

$$
\begin{gathered}
\dot{C}_{a}+i \omega_{a b} C_{a}=i\left[\Omega_{a b}^{\text {drive }}+\Omega^{\text {super }}\right] C_{b}, \\
\dot{C}_{c}+i \omega_{c b} C_{c}=i \Omega_{c b}^{\text {drive }} C_{b}, \\
\dot{C}_{b}=i\left[\Omega_{a b}^{\text {drive* }}+\Omega^{\text {super }}\right] C_{a}+i \Omega_{c b}^{\text {drive }^{*}} C_{c} .
\end{gathered}
$$

We assume that initially all atoms are in the ground state $b$. Coherence of the $a-b$ transition is

$$
\rho_{a b}=C_{a} C_{b}^{*}
$$

which yields

$$
\rho_{a b}^{\text {super }}=C_{a}^{\text {super }} C_{b}^{\text {drive* }}+C_{a}^{\text {drive }} C_{b}^{\text {super* }} .
$$

Equations (G8)-(G10) give the following equations of atomic evolution under the influence of the driving field:

$$
\begin{gathered}
\dot{C}_{a}^{\text {drive }}+i \omega_{a b} C_{a}^{\text {drive }}=i \Omega_{a b}^{\text {drive }} C_{b}^{\text {drive }}, \\
\dot{C}_{c}^{\text {drive }}+i \omega_{c b} C_{c}^{\text {drive }}=i \Omega_{c b}^{\text {drive }} C_{b}^{\text {drive }}, \\
\dot{C}_{b}^{\text {drive }}=i \Omega_{a b}^{\text {drive* }} C_{a}^{\text {drive }}+i \Omega_{c b}^{\text {drive* }} C_{c}^{\text {drive }},
\end{gathered}
$$

while for the quantities describing the high-frequency field, we find 


$$
\begin{gathered}
\dot{C}_{a}^{\text {super }}+i \omega_{a b} C_{a}^{\text {super }}=i \Omega^{\text {super }} C_{b}^{\text {drive }}+i \Omega_{a b}^{\text {drive }} C_{b}^{\text {super }}, \\
\dot{C}_{b}^{\text {super }}=i \Omega^{\text {super }} C_{a}^{\text {drive }}+i \Omega_{a b}^{\text {drive* }} C_{a}^{\text {super }} .
\end{gathered}
$$

Writing

$$
\begin{gathered}
\Omega^{\text {super }}=\Omega_{s} e^{-i \omega_{a b} t+i \omega_{a b} z / c}+\text { c.c. }, \\
\rho_{a b}^{\text {super }}+\text { c.c. }=\rho_{a b}^{s} e^{-i \omega_{a b} t+i \omega_{a b} z / c}+\text { c.c., } \\
C_{a}^{\text {super }}=C_{1 a}^{s} e^{-i \omega_{a b} t+i \omega_{a b} z / c}+C_{2 a}^{s} e^{i \omega_{a b} t-i \omega_{a b} z / c}, \\
C_{b}^{\text {super }}=C_{1 b}^{s} e^{-i \omega_{a b} t+i \omega_{a b} z / c}+C_{2 b}^{s} e^{i \omega_{a b} t-i \omega_{a b} z / c},
\end{gathered}
$$

where $\Omega_{s}, \rho_{a b}^{s}, C_{1 a}^{s}, C_{2 a}^{s}, C_{1 b}^{s}$, and $C_{2 b}^{s}$ are slowly varying functions as compared to the fast-oscillating exponentials, we obtain

$$
\rho_{a b}^{s}=C_{1 a}^{s} C_{b}^{\text {drive* }}+C_{1 b}^{s} C_{a}^{\text {drive* }}+C_{2 a}^{s *} C_{b}^{\text {drive }}+C_{2 b}^{s *} C_{a}^{\text {drive }} .
$$

Equations (G16) and (G17) yield

$$
\begin{gathered}
\dot{C}_{1 a}^{s}=i \Omega_{s} C_{b}^{\text {drive }}+i \Omega_{a b}^{\text {drive }} C_{1 b}^{s}, \\
\dot{C}_{2 a}^{s}+2 i \omega_{a b} C_{2 a}^{s}=i \Omega_{s}^{*} C_{b}^{\text {drive }}+i \Omega_{a b}^{\text {drive }} C_{2 b}^{s}, \\
\dot{C}_{1 b}^{s}-i \omega_{a b} C_{1 b}^{s}=i \Omega_{s} C_{a}^{\text {drive }}+i \Omega_{a b}^{\text {drive }} C_{1 a}^{s}, \\
\dot{C}_{2 b}^{s}+i \omega_{a b} C_{2 b}^{s}=i \Omega_{s}^{*} C_{a}^{\text {drive }}+i \Omega_{a b}^{\text {drive }} C_{2 a}^{s} .
\end{gathered}
$$

Equations (G22)-(G26) have to be supplemented by Maxwell's equation for the high-frequency field envelope $\Omega_{s}$,

$$
\left(c \frac{\partial}{\partial z}+\frac{\partial}{\partial t}\right) \Omega_{s}=i \Omega_{a}^{2} \rho_{a b}^{s},
$$

where $\Omega_{a}$ is the collective atomic frequency.

We assume that the driving field is weak and keep only terms of the proper order in $1 / \omega_{a b}$. Then, Eqs. (G24)-(G26) yield

$$
\begin{gathered}
C_{2 a}^{s} \approx \frac{C_{b}^{\text {drive }}}{2 \omega_{a b}} \Omega_{s}^{*}+i \frac{\dot{C}_{2 a}^{s}}{2 \omega_{a b}} \approx \frac{C_{b}^{\text {drive }}}{2 \omega_{a b}} \Omega_{s}^{*}+i \frac{C_{b}^{\text {drive }}}{4 \omega_{a b}^{2}} \dot{\Omega}_{s}^{*}, \\
C_{1 b}^{s} \approx-\frac{\Omega_{a b}^{\text {drive* }}}{\omega_{a b}} C_{1 a}^{s}, \\
C_{2 b}^{s} \approx \frac{\Omega_{a b}^{\text {drive* }}}{\omega_{a b}} C_{2 a}^{s} \approx \frac{\Omega_{a b}^{\text {drive }}}{2 \omega_{a b}^{2}} C_{b}^{\text {drive }} \Omega_{s}^{*} .
\end{gathered}
$$

Taking this into account, one can rewrite Eq. (G22) as

$$
\begin{aligned}
\rho_{a b}^{s}= & C_{1 a}^{s}\left(C_{b}^{\text {drive* }}-\frac{\Omega_{a b}^{\text {drive* }}}{\omega_{a b}} C_{a}^{\text {drive }^{*}}\right) \\
& +\frac{\left|C_{b}^{\text {drive }}\right|^{2}}{2 \omega_{a b}} \Omega_{s}-i \frac{\left|C_{b}^{\text {drive }}\right|^{2}}{4 \omega_{a b}^{2}} \dot{\Omega}_{s} .
\end{aligned}
$$

In this equation, one should take $\left|C_{b}^{\text {drive }}\right|^{2} \approx 1$. Thus, the two last terms in Eq. (G31) do not produce modulation, and hence, they can be omitted. Taking the time derivative of both sides of Eq. (G27), using Eqs. (G23), (G31), and (G13)-(G15), one can reduce the evolution equation for the high-frequency field envelope $\Omega_{s}$ to

$$
\begin{aligned}
& \left(\frac{\partial}{\partial t}+2 i \frac{\left|\Omega_{a b}^{\text {drive }}\right|^{2}}{\omega_{a b}}+i \frac{\left|\Omega_{c b}^{\text {drive }}\right|^{2}}{\omega_{c b}}+2 \frac{\Omega_{a b}^{\text {drive }} \dot{\Omega}_{a b}^{\text {drive* }}}{\omega_{a b}^{2}}\right) \\
& \times\left(c \frac{\partial}{\partial z}+\frac{\partial}{\partial t}\right) \Omega_{s}+\Omega_{a}^{2}\left(1-\frac{\left|\Omega_{a b}^{\text {drive }}\right|^{2}}{\omega_{a b}^{2}}-\frac{\left|\Omega_{c b}^{\text {drive }}\right|^{2}}{\omega_{c b}^{2}}\right. \\
& \left.-\frac{\Omega_{a b}^{\text {drive* }} \Omega_{a b}^{\text {drive* }}}{\omega_{a b}^{2}}\right) \Omega_{s}=0 .
\end{aligned}
$$

The unwanted ac Stark shift is compensated if we choose

$$
2 \frac{\left|\Omega_{a b}^{\text {drive }}\right|^{2}}{\omega_{a b}}+\frac{\left|\Omega_{c b}^{\text {drive }}\right|^{2}}{\omega_{c b}}=\text { const. }
$$

Let us assume that $\Omega_{a b}^{\text {drive }}$ is real and given by

$$
\Omega_{a b}^{\text {drive }}=\Omega_{d} \cos \left(\nu_{d} t-k_{d} z\right) .
$$

Then, if $\omega_{a b}=\omega_{c b}$, the time dependence of the ac Stark shift is compensated, provided that

$$
\Omega_{c b}^{\text {drive }}=\sqrt{2} \Omega_{d} \sin \left(\nu_{d} t-k_{d} z\right)
$$

For such parameters, Eq. (G32) becomes

$$
\begin{aligned}
\left(\frac{\partial}{\partial t}\right. & \left.+i \frac{2 \Omega_{d}^{2}}{\omega_{a b}}-\frac{\nu_{d} \Omega_{d}^{2}}{\omega_{a b}^{2}} \sin \left(2 \nu_{d} t-2 k_{d} z\right)\right)\left(c \frac{\partial}{\partial z}+\frac{\partial}{\partial t}\right) \Omega_{s} \\
& +\Omega_{a}^{2}\left(1-\frac{2 \Omega_{d}^{2}}{\omega_{a b}^{2}}\right) \Omega_{s}=0
\end{aligned}
$$

which yields an exponentially growing solution if $\nu_{d} \gtrsim \Omega_{a}$.

Our findings show that it is possible to compensate the time dependence of the ac Stark shift by the proper choice of the driving-field polarization. At the same time, the transition strength is still modulated by the driving field, which leads to the gain at high frequencies. The result remains valid for realistic atoms with many levels.

[1] M. O. Scully, E. Fry, C. H. R. Ooi, and K. Wodkiewicz, Directed Spontaneous Emission from an Extended Ensemble of $N$ Atoms: Timing Is Everything, Phys. Rev. Lett. 96, 010501 (2006).

[2] I.E. Mazets and G. Kurizki, Multiatom Cooperative Emission Following Single-Photon Absorption: DickeState Dynamics, J. Phys. B 40, F105 (2007). 
[3] A. A. Svidzinsky, J. Chang, and M. O. Scully, Dynamical Evolution of Correlated Spontaneous Emission of a Single Photon from a Uniformly Excited Cloud of N Atoms, Phys. Rev. Lett. 100, 160504 (2008).

[4] A. A. Svidzinsky and J. Chang, Cooperative Spontaneous Emission as a Many-Body Eigenvalue Problem, Phys. Rev. A 77, 043833 (2008).

[5] R. Friedberg and J. T. Manassah, Effects of Including the Counterrotating Term and Virtual Photons on the Eigenfunctions and Eigenvalues of a Scalar Photon Collective Emission Theory, Phys. Lett. A 372, 2514 (2008).

[6] D. Porras and J. Cirac, Collective Generation of Quantum States of Light by Entangled Atoms, Phys. Rev. A 78, 053816 (2008).

[7] M. O. Scully and A. A. Svidzinsky, The Super of Superradiance, Science 325, 1510 (2009).

[8] L. Pedersen and K. Mølmer, Few Qubit Atom-Light Interfaces with Collective Encoding, Phys. Rev. A 79, 012320 (2009).

[9] R. Röhlsberger, K. Schlage, B. Sahoo, S. Couet, and R. Rüffer, Collective Lamb Shift in Single-Photon Superradiance, Science 328, 1248 (2010).

[10] S. Prasad and R. Glauber, Coherent Radiation by a Spherical Medium of Resonant Atoms, Phys. Rev. A 82, 063805 (2010).

[11] E. M. Kessler, S. Yelin, M. D. Lukin, J. I. Cirac, and G. Giedke, Optical Superradiance from Nuclear Spin Environment of Single-Photon Emitters, Phys. Rev. Lett. 104, 143601 (2010).

[12] A. A. Svidzinsky, J. Chang, and M. O. Scully, Cooperative Spontaneous Emission of $N$ Atoms: Many-Body Eigenstates, the Effect of Virtual Lamb Shift Processes, and Analogy with Radiation of $N$ Classical Oscillators, Phys. Rev. A 81, 053821 (2010).

[13] R. Wiegner, J. von Zanthier, and G. S. Agarwal, QuantumInterference-Initiated Superradiant and Subradiant Emission from Entangled Atoms, Phys. Rev. A 84, 023805 (2011).

[14] T. Bienaimé, N. Piovella, and R. Kaiser, Controlled Dicke Subradiance from a Large Cloud of Two-Level Systems, Phys. Rev. Lett. 108, 123602 (2012).

[15] A. A. Svidzinsky, Nonlocal Effects in Single-Photon Superradiance, Phys. Rev. A 85, 013821 (2012).

[16] R.H. Dicke, Coherence in Spontaneous Radiation Processes, Phys. Rev. 93, 99 (1954).

[17] D. C. Burnham and R. Y. Chiao, Coherent Resonance Fluorescence Excited by Short Light Pulses, Phys. Rev. 188, 667 (1969).

[18] S. E. Harris, Electromagnetically Induced Transparency, Phys. Today 50, 36 (1997).

[19] O. Kocharovskaya, Amplification and Lasing without Inversion, Phys. Rep. 219, 175 (1992).

[20] M. O. Scully and M.S. Zubairy, Quantum Optics (Cambridge University Press, Cambridge, England, 1997).

[21] A.S. Zibrov, M. Lukin, D. Nikonov, L. Hollberg, M. Scully, V. Velichansky, and H. Robinson, Experimental Demonstration of Laser Oscillation without Population Inversion via Quantum Interverence in $R b$, Phys. Rev. Lett. 75, 1499 (1995).

[22] M. Abramowitz and I. A. Stegun, Handbook of Mathematical Functions (U.S. Government Printing
Office, 1964; reprinted by Dover, New York, 1965), Chap. 20.

[23] L. D. Landau and E. M. Lifschitz, Mechanics (Nauka Publishers, Moscow, 1988).

[24] R. W. Boyd, Nonlinear Optics (Academic Press, New York, 2003).

[25] X. Zhang and A. A. Svidzinsky, Superradiant Control of $\gamma$-ray Propagation by Vibrating Nuclear Arrays, Phys. Rev. A 88, 033854 (2013).

[26] H. Heffner and G. Wade, Gain, Band Width, and Noise Characteristics of the Variable-Parameter Amplifier, J. Appl. Phys. 29, 1321 (1958).

[27] P. K. Tien, Parametric Amplification and Frequency Mixing in Propagating Circuits, J. Appl. Phys. 29, 1347 (1958).

[28] S. A. Akhmanov and R. V. Khokhlov, Concerning One Possibility of Amplification of Light Waves, Zh. Eksp. Teor. Fiz. 43, 351 (1962) [Sov. Phys. JETP 16, 252 (1962)].

[29] R. H. Kingston, Parametric Amplification and Oscillation at Optical Frequencies, Proc. IRE 50, 472 (1962).

[30] N. M. Kroll, Parametric Amplification in Spatially Extended Media and Application to the Design of Tunable Oscillators at Optical Frequencies, Phys. Rev. 127, 1207 (1962).

[31] J. A. Glordmaine and R.C. Miller, Tunable Coherent Parametric Oscillation in $\mathrm{LiNbO}_{3}$ at Optical Frequencies, Phys. Rev. Lett. 14, 973 (1965).

[32] S. A. Akhmanov and R. V. Khokhlov, Parametric Amplifiers and Generators of Light, Usp. Fiz. Nauk 88, 439 (1966) [Sov. Phys. Usp. 9, 210 (1966)].

[33] S. E. Harris, Tunable Optical Parametric Oscillators, Proc. IEEE 57, 2096 (1969).

[34] R. L. Byer, "Optical Parametric Oscillators," in Treatise in Quantum Electronics, 1B, edited by H. Rabin and C. L. Tang (Academic, New York, 1975), p. 587.

[35] C.S. Hsu, On the Parametric Excitation of a Dynamic System Having Multiple Degrees of Freedom, J. Appl. Mech. 30, 367 (1963).

[36] A. H. Nayfeh and D. T. Mook, Parametric Excitations of Linear Systems Having Many Degrees of Freedom, J. Acoust. Soc. Am. 62, 375 (1977).

[37] A.H. Nayfeh, Nonlinear Interactions: Analytical, Computational and Experimental Methods (Wiley, New York, 2000).

[38] C. C. Chen and M. K. Yen, Parametric Instability of a Cantilevered Column under Periodic Loads in the Direction of the Tangency Coefficient, J. Sound Vib. 183, 253 (1995).

[39] G. Cederbaum, Dynamic Instability of Shear Deformable Laminated Plates, AIAA J. 29, 2000 (1991).

[40] F. C. Fu and S. Nemat-Nasser, Coincidence Problem and Combination Resonance, AIAA J. 15, 1785 (1977).

[41] E. Mettler, "Stability and Vibration Problems of Mechanical Systems under Harmonic Excitation," Dynamic Stability of Structures, edited by G. Herrmann (Pergamon Press, New York, 1967), pp. 169-188.

[42] A. A. Mailybayev and A.P. Seyranian, Parametric Resonance in Systems with Small Dissipation, J. Appl. Math. Mech. 65, 755 (2001).

[43] J.G. Vioque, A.R. Champneys, and M. Truman, Difference Combination Parametric Resonance; 
Application to the Garden Hose Problem, Bol. Soc. Esp. Mat. Apl. 51, 63 (2010).

[44] L. R. Hunter, D. Krause, S. Murthy, and T. Sung, Precision Measurement of the Stark Shift of the Cesium D Lines, Phys. Rev. A 37, 3283 (1988).
[45] M. Kawamura, W.-G. Gin, N. Takahashi, and T. Minowa, Stark Effect of the Rubidium D2 Line Studied by HighResolution Laser Spectroscopy, J. Phys. Soc. Jpn. 78, 124301 (2009). 